\title{
SPREADING OF SINGULARITIES AT THE BOUNDARY IN SEMILINEAR HYPERBOLIC MIXED PROBLEMS II: CROSSING AND SELF-SPREADING
}

\author{
MARK WILLIAMS
}

\begin{abstract}
The creation of anomalous singuiarities in solutions to nonlinear hyperbolic equations due to crossing or self-spreading in free space is by now rather well understood. In this paper we study how anomalous singularities are produced in mixed problems for semilinear wave equations $\square u=f(u)$ on the half-space $\mathbf{R}_{+}^{n+1}, u \in H_{\mathrm{loc}}^{s}, s>(n+2) / 2$, due to crossing and self-spreading at boundary points. Several phenomena appear in the problems considered here which distinguish spreading at the boundary from spreading in free space: (1) Anomalous singularities of strength $\sim 2 s-n / 2$ can arise when incoming singularity-bearing rays cross or self-spread at a point on the boundary. A consequence of this, announced in [14], is that the analogue of Beals's $3 \mathrm{~s}$ theorem fails for reflection in second-order mixed problems. Although $H^{r}$ regularity for $r<\sim 3 s-n$ propagates along null bicharacteristics in free space, for $r>\sim 2 s-n / 2$ it does not in general reflect. (2) For nonlinear wave equations in free space, anomalous singular support is never produced by the interaction of fewer than three bicharacteristics, unless self-spreading occurs. However, anomalous singularities can arise when a pair of rays cross at a boundary point. (3) Suppose $\square u=u^{2}$ and $u \in C^{\infty}$ on the boundary. For certain choices of initial data, anomalous singularities of strength $\sim 2 s-n / 2$ arise at the boundary from three sources: interactions of incoming rays with incoming rays, incoming rays with reflected rays, and reflected rays with reflected rays. Singularities produced by the incoming-reflected interactions differ in sign from and are strictly weaker than the other two types, so some cancellations occur. As the incoming rays approach being gliding rays, the difference in strength decreases and hence the cancellations become increasingly significant.
\end{abstract}

\section{INTRODUCTION AND STATEMENT OF RESULTS}

This paper is a continuation of the study begun in [14] of how singularities spread at the boundary in mixed problems for semilinear strictly hyperbolic equations. Whereas [14] was mainly concerned with propagation of regularity, here our goal is to determine the strength and location of anomalous singularities and to analyze the role of the boundary in their creation. Together, these two papers represent an attempt to determine to what extent results analogous to those on spreading for second-order equations in free space (M. Beals [1, 2]) can be proved for boundary problems. We refer to the introduction of [14] for a

Received by the editors October 13, 1987.

1980 Mathematics Subject Classification (1985 Revision). Primary 58G17, 35L70.

Partially supported by NSF Grant DMS-8701654. 
brief survey of recent work on nonlinear propagation theory and for additional references.

We shall focus here on two problems. For $n \geq 2$ let $\mathbf{R}_{+}^{n}=\{(x, y): x>0\}$, and set $\Omega=(-\infty, \infty) \times \overline{\mathbf{R}}_{+}^{n}, \Omega_{T}=(-T, T) \times \overline{\mathbf{R}}_{+}^{n}$, and $b \Omega_{T}=\Omega_{T} \cap\{x=0\}$. Consider a function $u(t, x, y) \in H_{\mathrm{loc}}^{s}\left(\Omega_{T}\right), s>(n+2) / 2$, which satisfies

$$
\square u=\left(D_{t}^{2}-\Delta\right) u=\beta(t) f(u),\left.\quad u\right|_{b \Omega_{T}} \in C^{\infty},
$$

where $\beta(t) \in C_{0}^{\infty}$ has $\operatorname{supp} \beta \subset\{|t|<\delta\}, \delta<T$. With $z=(t, x, y)$ and using $\zeta=(\tau, \xi, \eta)$ to denote the dual variables, we can now state:

Problem A (Crossing). Let $\Gamma_{1}=\left\{\left(z_{1}(t), \zeta_{1}\right)\right\}, \Gamma_{2}=\left\{\left(z_{2}(t), \zeta_{2}\right)\right\}$ be incoming null bicharacteristics such that $\left\{z_{1}(t)\right\},\left\{z_{2}(t)\right\}$ strike $b \Omega_{T}$ transversely when $t=0$ and cross there (i.e., $\left.z_{1}(0)=z_{2}(0) \in b \Omega_{T}, \zeta_{1} \neq \pm \zeta_{2}\right)$. Suppose that WF $\left.u\right|_{t<-\delta}=\left.\left.\Gamma_{1}\right|_{t<-\delta} \cup \Gamma_{2}\right|_{t<-\delta}$ (where by $\left.\Gamma_{i}\right|_{t<-\delta}$ we mean $\left\{\left(z_{i}(t), r \zeta_{i}\right): r>0\right.$, $t<-\delta\})$. Determine the location and strength of the anomalous singularities that may arise.

Problem B (Self-Spreading). Let $\Gamma_{ \pm}=\{(z(t), \pm \zeta)\}$ be incoming null bicharacteristics such that $\{z(t)\}$ strikes $b \Omega_{T}$ transversely when $t=0$. Suppose that $\left.W F u\right|_{t<-\delta}=\left.\left.\Gamma_{+}\right|_{t<-\delta} \cup \Gamma_{-}\right|_{t<-\delta}$. Determine the location and strength of the anomalous singularities that may arise.

It is known that certain hypotheses on the nature of the incoming singularities (e.g., conormality with respect to characteristic surfaces) can prevent or greatly inhibit the appearance of anomalous singularities in mixed problems $[4,5,10]$. Papers [7 and 14] provide two constraints that apply regardless of the structure of the incoming singularities. First, anomalous singularities in Problems A and B can have strength at most $\sim 2 s-n / 2$ (see Theorem 2.7). Moreover, one can use Theorem 1.7 of [14] to identify regions, depending only on $\zeta_{1}$ and $\zeta_{2}$, into which singularities cannot spread in Problem A (see Remark 1.10). Our main result, Theorem 1.9, shows that for certain choices of $\beta(t), f(u)$, and incoming singularities, the "worst" that could possibly happen, in view of the above constraints, does happen. In Problem A singularities of strength $\sim 2 s-n / 2$ appear everywhere on that portion of the forward half light-cone with vertex at $z_{1}(0)=z_{2}(0)$ which is not ruled out; in Problem B singularities of strength $\sim 2 s-n / 2$ appear everywhere on the forward half light-cone with vertex at $z(0)$.

The reader familiar with spreading in free space will notice in the above paragraph two new phenomena due to the presence of the boundary. For secondorder strictly hyperbolic equations in free space, anomalous singular support is never produced by the interaction of fewer than three bicharacteristics, unless self-spreading occurs [1, 2]. Furthermore, anomalous singularities (in such problems) due to either crossing or self-spreading in free space can have strength at most $\sim 3 s-n[2,3]$. The appearance of singularities of strength $\sim 2 s-n / 2$ in Problems A and B implies that the analogue of Beals's $3 s$ theorem does not hold for reflection at the boundary in second-order mixed problems. 


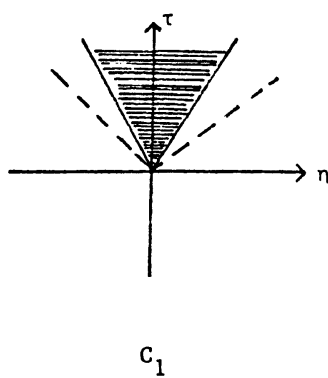

(a)

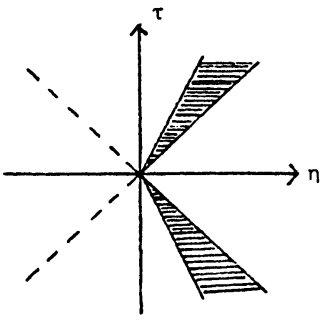

$\mathrm{c}_{2}$

(b)

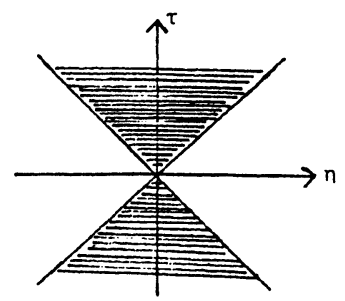

$\mathrm{C}_{3}$

(c)

FIGURE 1 . The case $n=2$

We proceed to state the main results. Consider the mixed problem on $\Omega_{T}$

$$
\begin{aligned}
& \square u=\beta u^{2},\left.\quad u\right|_{b \Omega_{T}} \in C^{\infty}, \\
& \left.u\right|_{t=-T}=w_{0},\left.\quad u_{t}\right|_{t=-T}=w_{1},
\end{aligned}
$$

where $\beta \in C_{0}^{\infty}$ is chosen so that $\operatorname{supp} \beta \subset\{|t|<\delta\}$ for $\delta<T, \widehat{\beta} \geq 0$, and $\widehat{\beta}(0)>0 . w_{0}$ and $w_{1}$ are taken to be the Cauchy data at $t=-T$ of a function $w(t, x, y) \in H_{\mathrm{loc}}^{s}\left(R^{n+1}\right), s>(n+2) / 2$, defined as follows.

For any $\omega \in S^{n-1}$ denote by $f_{\omega}(x, y)$ an element of $H^{s}\left(R^{n}\right)$ with the properties, for $\rho>1$ :

(1.3) (a) $W F \quad f_{\omega}=\{(0, r \omega): r>0\}$.

(b) $0 \leq \hat{f}_{\omega}(\xi, \eta) \leq C\langle\xi, \eta\rangle^{-(s+n / 2+a(\rho))}$, where $a(\rho)>0, a(\rho)=O(\rho-1)$.

(Here $\langle\xi, \eta\rangle=\left(1+|\xi, \eta|^{2}\right)^{1 / 2}$.)

(c) $|(\xi, \eta)-| \xi, \eta|\omega| \leq|\xi, \eta|^{1 / \rho}$ implies $\hat{f}_{\omega}(\xi, \eta) \geq C^{\prime}\langle\xi, \eta\rangle^{-(s+n / 2+a(\rho))}$.

Functions satisfying (1.3)(a)-(c) are constructed in [1].

Now take $\omega_{1}, \omega_{2}, \widetilde{\omega} \in S^{n-1}$ equal to $(1 / \sqrt{2}, 1 / \sqrt{2}, 0),(1 / \sqrt{2},-1 / \sqrt{2}, 0)$, $(1,0)$, respectively, and define $f_{1}, f_{2}, g \in H^{s}\left(R^{n}\right)$ by taking $\omega$ in (1.3) equal

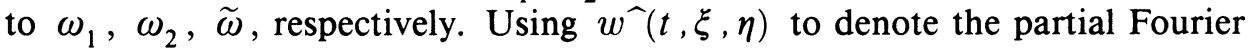
transform, we shall consider three possibilities for $w$ :
(a) $w^{-}(t, \xi, \eta)=e^{i t|\xi, \eta|}\left[\hat{f}_{1}(\xi, \eta)+\hat{f}_{2}(\xi, \eta)\right]$,
(b) $w^{\frown}(t, \xi, \eta)=e^{i t|\xi, \eta|} \hat{f}_{1}(\xi, \eta)+e^{-i t|\xi, \eta|} \hat{f}_{2}(-\xi,-\eta)$,
(c) $w^{\wedge}(t, \xi, \eta)=e^{i t|\xi, \eta|} \hat{g}(\xi, \eta)+e^{-i t|\xi, \eta|} \hat{g}(-\xi,-\eta)$.

Note that $\square w=0$ and that $W F w$ in cases $a, b, c$ is respectively equal to $r>0\}$,

(a) $\bigcup_{i=1,2}\left\{\left(t,-t \omega_{i}, r, r \omega_{i}\right): t \in \mathbf{R}, r>0\right\}$,

(b) $\left\{\left(t,-t \omega_{1}, r, r \omega_{1}\right): t \in \mathbf{R}, r>0\right\} \cup\left\{\left(t,-t \omega_{2},-r,-r \omega_{2}\right): t \in \mathbf{R}\right.$,

(c) $\{(t,-t \widetilde{\omega}, r, r \widetilde{\omega}): t \in \mathbf{R}, r \in \mathbf{R} \backslash 0\}$.

Next we define the sets that will be seen to carry anomalous singularities. First, for $\omega \in S^{n-1}$ let $K_{ \pm}^{\omega}$ be the rays through $\pm(1, \omega)$ in $R^{n+1} \backslash 0((\tau, \xi, \eta)$ space) and set (with notation borrowed from [2]) 
(1.6) (a) $B^{\omega_{1}, \omega_{2}}=\overline{K_{+}^{\omega_{1}}+K_{+}^{\omega_{2}}}\left(\right.$ closure in $\left.R^{n+1} \backslash 0\right)$,

(b) $B^{\omega_{1},-\omega_{2}}=\overline{K_{+}^{\omega_{1}}+K_{-}^{\omega_{2}}}$,

(c) $B^{\tilde{\omega}}=\{$ tangent plane to char $\square$ at $\pm(1, \tilde{\omega})\}$.

Using $\pi$ to denote the projection $(\tau, \xi, \eta) \rightarrow(\tau, \eta)$, we set
(a) $C_{1}=\pi B^{\omega_{1}, \omega_{2}} \cap\left\{\tau^{2} \geq|\eta|^{2}\right\}=\pi B^{\omega_{1}, \omega_{2}}$,
(b) $C_{2}=\pi B^{\omega_{1},-\omega_{2}} \cap\left\{\tau^{2} \geq|\eta|^{2}\right\}$,
(c) $C_{3}=\pi B^{\tilde{\omega}} \cap\left\{\tau^{2} \geq|\eta|^{2}\right\}=\left\{\tau^{2} \geq|\eta|^{2}\right\}$.

Finally, for $i=1,2,3$ we define

(1.8) $\Lambda_{i}=\left\{\lambda=(t, x, y, \tau, \xi, \eta): t>0,(t, x, y) \in \stackrel{\circ}{\Omega}_{T}\right.$, and $\lambda$ lies on the outgoing (i.e., $d x / d t>0)$ null bicharacteristic that passes over $(0,(\tau, \eta))$, for some $\left.(\tau, \eta) \in \stackrel{\circ}{C}_{i}\right\}$.

(Let $\pi_{1}$ be the projection $(t, x, y, \tau, \xi, \eta) \rightarrow(t, x, y)$. Then in Figure 2 $\pi_{1} \Lambda_{i}$ is the shaded region in each case.) If we set $w_{0}, w_{1}$ in (1.2) equal to $\left.w\right|_{t=-T, x \geq 0},\left.w_{t}\right|_{t=-T, x \geq 0}$ respectively, the existence of a solution $u \in H^{s}\left(\Omega_{T}\right)$ follows from a classical iteration argument provided $T$ is small enough, as we shall assume.

Theorem 1.9. Fix $\varepsilon>0$ arbitrarily small.

(a) (Crossing) Define $w_{0}, w_{1}$ in (1.2) using $w$ as in (1.4)(a). Then if $\rho$ in $(1.3)(\mathrm{c})$ is taken close enough to 1 , the solution $u \in H^{s}\left(\Omega_{T}\right)$ of (1.2) has singularities of strength $2 s-n / 2+2+\varepsilon$ throughout $\Lambda_{1} ;$ that is, $u \notin$ $H^{2 s-n / 2+2+\varepsilon}(\lambda)$ for all $\lambda \in \Lambda_{1}$. (See Figure 2a.)

(b) (Crossing) The statement is the same as in part (a), except that $w$ as in (1.4)(b) is used, and $\Lambda_{2}$ is substituted for $\Lambda_{1}$ (Figure 2b).

(c) (Self-spreading) The statement is the same as in part (a), except that $w$ as in $(1.4)(\mathrm{c})$ is used, and $\Lambda_{3}$ is substituted for $\Lambda_{1}$ (Figure 2c).

Remark 1.10. Note that in parts (a) and (b) of Theorem 1.9 the singularitybearing characteristics are the same in $t<0$, and the regions carrying anomalous singularities are 2-dimensional sheets contained in the forward half lightcone. Theorem 1.7 of [14] implies that in $t>0, x>0 u$ is $C^{\infty}$ outside those

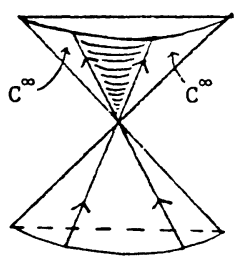

(a)

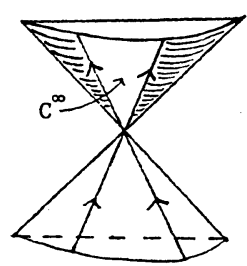

(b)

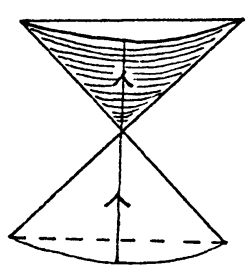

(c)

FIGURE 2. The case $n=2$.

On the shaded portions $u \notin H^{2 s-n / 2+2+\varepsilon}$ 
sheets. In (c) new singularities appear throughout the $n$-dimensional forward half light-cone.

In (b) new singularities are also carried by a pair of forward gliding rays (since sing supp $u$ is closed), while in (c) they appear on all forward gliding rays through the origin.

Outline of the proof of Theorem 1.9. Here we shall establish some notation, make reductions that will be used throughout the paper, and point out why anomalous singularities of strength $\sim 2 s-n / 2$ should be expected in the presence of a boundary.

Following an idea like that used by Beals $[1,2]$ in free space, we first write

$$
u=v+R \beta u^{2}
$$

where $v \in H_{\text {loc }}^{S}(\Omega)$ satisfies (in $\Omega$ )

$$
\square v=0,\left.\quad v\right|_{b \Omega} \in C^{\infty},\left.\quad v\right|_{t=-T}=w_{0},\left.\quad v_{t}\right|_{t=-T}=w_{1},
$$

and $R$ is the forward solution operator such that for any $U \in L_{\text {loc }}^{2}(\Omega)$ supported in $t>-\delta, R U$ satisfies (in $\Omega$ )

$$
\square R U=U,\left.\quad R U\right|_{b \Omega}=0, \quad R U=0 \text { in } t<-\delta .
$$

Next rewrite $u$ as $u=v+R \beta v^{2}+R \beta\left(u^{2}-v^{2}\right)$. The plan is to find singularities of strength $2 s-n / 2+2+\varepsilon$ in $R \beta v^{2}$ on $\Lambda_{i}$ and then show that any singularities of the remainder $R \beta\left(u^{2}-v^{2}\right)$ must be strictly weaker there, completing the proof.

Singularities of $R \beta v^{2}$. Notice that $v$ is $\left(\bmod C^{\infty}\right)$ simply the restriction to $x>0$ of the function in $H_{\text {loc }}^{s}\left(\mathbf{R}^{n+1}\right)$, which we will also call $v$, given by

$$
\widehat{v}(t, \xi, \eta)=\widehat{w}(t, \xi, \eta)-\widehat{w}(t,-\xi, \eta) .
$$

Henceforth we will write $v=v_{a}-v_{b}$ where $v_{a}$ and $v_{b}$, whose singularities lie respectively on incoming and outgoing rays, are defined by (1.14). We begin to see here an interesting feature of spreading at the boundary; namely, singularities of $R \beta v^{2}$ (and hence anomalous singularities of $u$ ) can arise from three kinds of interactions-incoming rays with incoming rays, incoming rays with outgoing rays, and outgoing rays with outgoing rays. These interactions make contributions not all with the same sign, so to detect singularities in $R \beta v^{2}$ it must be shown that the cancellations are not complete. To make this precise, we first write

$$
R \beta v^{2}=\left.\left(E \beta v^{2}\right)\right|_{x>0}-C\left(\left.\left(E \beta v^{2}\right)\right|_{b \Omega}\right),
$$

where $E$ is the solution operator such that for $U \in L_{\text {loc }}^{2}\left(\mathbf{R}^{n+1}\right)$ supported in $t>-\delta, E U$ satisfies (in $\mathbf{R}^{n+1}$ )

$$
\square E U=U, \quad E U=0 \quad \text { in } t<-\delta,
$$


and $C$ is such that, for $V \in H_{\mathrm{loc}}^{1}(b \Omega)$ supported in $t>-\delta, C V$ satisfies (in $\Omega$ )

$$
\square C V=0,\left.\quad(C V)\right|_{b \Omega}=V, \quad C V=0 \quad \text { in } t<-\delta .
$$

For $V \in H_{\mathrm{loc}}^{1}(b \Omega)$ known only to satisfy sing supp $V \subset\{t>-\delta\}$, we shall also denote by $C$ an operator such that

$$
\left(1.17^{\prime}\right) \quad \square C V=0,\left.\quad(C V)\right|_{b \Omega}=V, \quad \text { sing supp } C V \subset\{t>-\delta\} .
$$

The term $\left.\left(E \beta v^{2}\right)\right|_{x>0}$ in (1.15) cannot possibly contribute anomalous singular support to $u$, simply because

$$
\pi_{2} W F v^{2} \cap \operatorname{char} \square=\pi_{2} W F v \cap \operatorname{char} \square\left(\pi_{2}:(t, x, y, \tau, \xi, \eta) \rightarrow(\tau, \xi, \eta)\right)
$$

in each of the three cases. For example, suppose $w$ as in (1.4)(a) is used to define $v_{a}$ and $v_{b}$, and write $v_{a}=v_{a 1}+v_{a 2}, v_{b}=v_{b 1}+v_{b 2}$, where $\widehat{v_{a i}}=e^{i t|\xi, \eta|} \hat{f}_{i}(\xi, \eta), \widehat{v_{b i}}=e^{i t|\xi, \eta|} \hat{f}_{i}(-\xi, \eta)$. Then one has $\pi_{2} W F v_{a 1}^{2}=K_{+}^{\omega_{1}}$, $\pi_{2} W F v_{a 1} v_{a 2} \subset B^{\omega_{1}, \omega_{2}}$ (which satisfies $B^{\omega_{1}, \omega_{2}} \cap$ char $\square=K_{+}^{\omega_{1}} \cup K_{+}^{\omega_{2}}$ ), and similarly for the other terms in $v^{2}$. When $w$ as in $(1.4)(\mathbf{b})$ is used, the argument is essentially the same. The third case is more subtle, for then $\pi_{2} W F v_{a 1}$ and $\pi_{2} W F v_{a 2}$ are antipodal (similarly for $v_{b 1}, v_{b 2}$ ). By a result of $\mathrm{M}$. Beals [2, Corollary 1.6], $\pi_{2} W F v_{a 1} v_{a 2} \subset B^{\tilde{\omega}}$ and $\pi_{2} W F v_{b 1} v_{b 2} \subset B^{-\widetilde{\omega}}$. Since the other terms resemble those in the first two cases, this implies that again $\pi_{2} W F v^{2}$ contains no new characteristic directions. Thus in each case we have reduced to considering $C\left(\left.\left(E \beta v^{2}\right)\right|_{b \Omega}\right)$. It should be noted that the same argument gives the main reason why anomalous singularities of strength $\sim 2 s-n / 2$ never appear in solutions to $\square u=f(u)$ due to spreading in free space.

Since $v^{2}=\left(v_{a}^{2}-v_{a} v_{b}\right)+\left(v_{b}^{2}-v_{a} v_{b}\right)$ we can reduce further, as will be clear from the proofs of $\S \S 3$ and 4 , to considering

$$
C\left(\left.\left(E \beta v_{a}^{2}\right)\right|_{b \Omega}\right)-C\left(\left.\left(E \beta v_{a} v_{b}\right)\right|_{b \Omega}\right) .
$$

Writing

(1.19) $v_{a}^{2}=v_{a 1}^{2}+2 v_{a 1} v_{a 2}+v_{a 2}^{2}, \quad v_{a} v_{b}=v_{a 1} v_{b 1}+v_{a 1} v_{b 2}+v_{a 2} v_{b 1}+v_{a 2} v_{b 2}$,

we examine first

$$
C\left(\left.\left(E \beta v_{a 1} v_{a 2}\right)\right|_{b \Omega}\right)-C\left(\left.\left(E \beta v_{a 1} v_{b 2}\right)\right|_{b \Omega}\right),
$$

supposing again that $w$ as in (1.4)(a) is used to define $v$. We claim that each term in (1.20) has singularities of strength $\sim 2 s-n / 2$ on $\Lambda_{1}$.

To see why, begin by recalling that $\pi_{2} W F \beta v_{a 1} v_{a 2} \subset B^{\omega_{1}, \omega_{2}}$. In $\S 3$ we will show that, in fact, $\beta v_{a 1} v_{a 2}$ and therefore also $E \beta v_{a 1} v_{a 2}$ have (microlocal) singularities of strength $\sim 2 s-n / 2$ throughout $\stackrel{\circ}{B}^{\omega_{1}, \omega_{2}}$ over $(t, x, y)=0$. Hence one expects $\left.\left(E \beta v_{a 1} v_{a 2}\right)\right|_{b \Omega}$ to have singularities of strength $\sim 2 s-n / 2$ on $\stackrel{\circ}{C}_{1}=\pi \stackrel{\circ}{B}^{\omega_{1}, \omega_{2}}$ over $0 \in b \Omega$. Since $C_{1} \subset\left\{\tau^{2}>|\eta|^{2}\right\}$, all of these singularities 
should be picked up and propagated by the operator $C$, leading to singularities of strength $\sim 2 s-n / 2$ throughout $\Lambda_{1}$. The second term in (1.20) also has such singularities on $\Lambda_{1}$ by a similar argument (note that $\pi_{2} W F \beta v_{a 1} v_{b 2}=B^{\omega_{1}, \omega_{2}^{*}}$, where $\omega_{2}^{*}=(-1 / \sqrt{2},-1 / \sqrt{2}, 0)$, and $\pi B^{\omega_{1}, \omega_{2}^{*}}$ also equals $\left.C_{1}\right)$, but we will show that these are actually strictly weaker (Lemma 3.18).

$C\left(\left.\left(E \beta v_{a 1} v_{a 2}\right)\right|_{b \Omega}\right)-C\left(\left.\left(E \beta v_{a 2} v_{b 1}\right)\right|_{b \Omega}\right)$, by the same analysis, contributes singularities of strength $\sim 2 s-n / 2$, and it is clear that these cannot cancel those of (1.20) since the same term dominates in both cases. The analysis of $R \beta v^{2}$ in part (a) of Theorem 1.9 is concluded by observing that the remaining terms in (1.19) clearly contribute nothing anomalous to $C\left(\left.\left(E \beta v^{2}\right)\right|_{b \Omega}\right)$.

In $\S \S 3$ and 4 , using a well-known result on reflection of $W F_{b}$, we replace the technically inconvenient operator $E$ in the above argument by multipliers like $H(\tau, \xi, \eta) /\left(\tau^{2}-\left(\xi^{2}+|\eta|^{2}\right)\right)$, where $H$ is the characteristic function of a small conic neighborhood of, for example, a point in $\stackrel{\circ}{B}^{\omega_{1}, \omega_{2}}$ (see Lemma 3.3).

The analysis of $R \beta v^{2}$ in parts (b) and (c) of Theorem 1.9 follows the same outline. For example, in part (c) we show first that $\beta v_{a 1} v_{a 2}$ has singularities of strength $\sim 2 s-n / 2$ throughout $B^{\tilde{\omega}} . B^{\tilde{\omega}}$ projects onto the whole of $(\tau, \eta)$ space, but the only singularities (in $\left.\left.\left(E \beta v_{a 1} v_{a 2}\right)\right|_{b \Omega}\right)$ that $C$ can propagate are those at points $(0,(\tau, \eta))$ with $\tau^{2} \geq|\eta|^{2}$. This explains why $C_{3}$ is chosen as in $(1.7)(\mathrm{c})$.

We wish to emphasize something already indicated in the discussion of (1.20). In each of the three cases of Theorem 1.9:

(1.21) The anomalous singularities on a fixed ray in $\Lambda_{i}$ produced by interactions of incoming rays with incoming rays $\left(v_{a}, v_{a}\right)$, or by interactions of outgoing rays with outgoing rays $\left(v_{b}, v_{b}\right)$, are stronger than those produced by incomingoutgoing interactions $\left(v_{a}, v_{b}\right)$ (see Remark 3.26).

The remainder $R \beta\left(u^{2}-v^{2}\right)$. By (1.11) we have that $u^{2}-v^{2}=2 v R \beta u^{2}+$ $\left(R \beta u^{2}\right)^{2}$, so we must consider two terms, $R \beta\left(v R \beta u^{2}\right)$ and $R \beta\left(R \beta u^{2}\right)^{2}$. Once a careful estimate of the regularity of $v^{2}$ is obtained (Lemmas 3.28 and 4.21), the second term can be handled using some of the $H^{t, t^{\prime}}$ microlocal algebra and propagation results of [14], but those results alone do not quite yield sufficient regularity of $R \beta\left(v R \beta u^{2}\right)$ on $\Lambda_{i}$. In order to find the extra smoothness needed, we extend certain microlocal pieces $U_{i}$ of $R \beta u^{2}$ across the boundary as solutions of wave equations in free space (Lemmas 3.36 and 4.38). This allows us to improve estimates like $\langle\tau, \xi, \eta\rangle^{s+1} \widehat{\phi R \beta u^{2}} \in L^{2}$ to estimates like $\langle\tau-|\xi, \eta|\rangle\langle\tau, \xi, \eta\rangle^{s+1} \widehat{U}_{i} \in L^{2}$, and with these the proof that $R \beta\left(u^{2}-v^{2}\right)$ does not cancel the singularities of $R \beta v^{2}$ can be completed. We remark that in the extension argument, the fact that the incoming singularities lie on rays that meet $b \Omega$ transversely plays an important role.

The plan of the paper is as follows. In $\S 2$ we collect results from [14] (and elsewhere) that are needed in the rest of the paper. In $\S 3$ parts (a) and (b) of 
Theorem 1.9 are proved, and in $\S 4$ we prove part (c). We refer to the introductory paragraph of $\S 4$ for some comments on the main technical differences between these two sections. In $\S 5$ two open problems are described.

A brief outline of the argument in the case of part (a) of Theorem 1.9 was included in the final section of [14].

Remark. In mixed problems for more general strictly hyperbolic equations $\mathrm{Pu}=$ $\beta f(u)$, anomalous singularities of strength $\sim 2 s-n / 2$ should be expected to arise by a process similar to that in the case $\square u=\beta u^{2}$. For example, suppose $f(u)=a_{2} u^{2}+a_{3} u^{3}+\cdots,\left.u\right|_{b \Omega_{T}} \in C^{\infty}$, and that the initial data are as in Theorem 1.9(a). Then $u$ can be written $u=v+R \beta f(v)+R \beta(f(u)-f(v))$, with $v=v_{a}-v_{b}, v_{a}=v_{a 1}+v_{a 2}, v_{b}=v_{b 1}+v_{b 2}$ as before. Consider the term $a_{m} R \beta v^{m}$ in $R \beta f(v) . v^{m}$ is a sum of terms like $C v_{a 1}^{m_{1}} v_{a 2}^{m_{2}} v_{b 1}^{m_{3}} v_{b 2}^{m_{4}}$. Let us write this as $C W$. If $m_{1}=m_{3}=0$ or $m_{2}=m_{4}=0, C W$ contributes nothing anomalous to $R \beta v^{m}$. In any other case $W$ has singularities of strength $\sim 2 s-n / 2$ (in addition to other singularities which can be as weak as $\sim 4 s$ ) in $(\tau, \xi, \eta)$-directions that project into $\stackrel{\circ}{C}_{1} \subset\left\{\tau^{2}>|\eta|^{2}\right\}$. By the argument in the case $\square u=\beta u^{2}$, these singularities give rise to anomalous singularities of strength $\sim 2 s-n / 2$ in $R \beta W$.

Acknowledgment. The influence of the work of Michael Beals on this paper is obvious, even in the title. I wish to thank him especially for conversations in which he suggested that anomalous singularities of strength $\sim 2 s-n / 2$ should be expected in second-order mixed problems.

\section{2. $H^{t, t^{\prime}}$ ALGEBRA AND PROPAGATION THEOREMS}

Here we collect for easy reference some information that will be needed in $\S \S 3$ and 4.

As before let $z=(t, x, y) \in \mathbf{R}^{n+1}, \zeta=(\tau, \xi, \eta)$, and $\Omega=\{(t, x, y): x \geq$ $0\}$. Sometimes we shall also set $z^{\prime}=(t, y)$ and $\zeta^{\prime}=(\tau, \eta)$. With $\langle\zeta\rangle=$ $\left(1+|\zeta|^{2}\right)^{1 / 2}, H^{t, t^{\prime}}\left(\mathbf{R}^{n+1}\right)$ is the space of tempered distributions $u$ such that $\hat{u}(\zeta) \in L_{\text {loc }}^{2}$ and $\langle\zeta\rangle^{t}\left\langle\zeta^{\prime}\right\rangle^{t^{\prime}} \hat{u} \in L^{2}\left(\mathbf{R}^{n+1}\right) . \quad H_{\mathrm{loc}}^{t, t^{\prime}}\left(\mathbf{R}^{n+1}\right)$ is the corresponding local space, and $H_{\text {loc }}^{t, t^{\prime}}(\Omega)$ the space of restrictions to $x>0$ of elements of $H_{\mathrm{loc}}^{t, t^{\prime}}\left(\mathbf{R}^{n+1}\right)$. Denote by $T^{m}(\Omega)$ the set of properly supported tangential pseudodifferential operators $\phi\left(z, D^{\prime}\right): H_{\mathrm{loc}}^{t, t^{\prime}}(\Omega) \rightarrow H_{\mathrm{loc}}^{t, t^{\prime}-m}(\Omega)$ associated to symbols $\phi\left(z, \zeta^{\prime}\right)$ of degree $m$. For $u \in H_{\text {loc }}^{t,-\infty}(\Omega)$ we define $\widetilde{W F}_{t, t^{\prime}} u \subset$ $\left(T^{*} b \Omega \backslash 0\right) \cup\left(T^{*} \stackrel{\circ}{\Omega} \backslash 0\right)$ as follows. In $T^{*} \stackrel{\circ}{\Omega} \backslash 0, \widetilde{W F}_{t, t^{\prime}} u=W F_{t+t^{\prime}} u$, where for any $r \in \mathbf{R}, W F_{r}$ is the usual measure of microlocal $H^{r}$ singularities. If $\sigma \in T^{*} b \Omega \backslash 0, \sigma \notin \widetilde{W F}_{t, t^{\prime}} u$ if and only if for some $\phi\left(z, D^{\prime}\right) \in T^{0}(\Omega)$, elliptic at $\sigma$, one has $\phi u \in H_{\text {loc }}^{t, t^{\prime}}(\Omega)$. In this case we write $u \in \widetilde{H}^{t, t^{\prime}}(\sigma)$. When $t^{\prime}=0$ instead of $\widetilde{W F}_{t, 0}, \widetilde{H}^{t, 0}$ we will usually write $\widetilde{W F}_{t}, \widetilde{H}^{t}$. 
Remark 2.1. In order to show that a given $u \in H_{\mathrm{loc}}^{t,-\infty}(\Omega)$ satisfies $u \in \widetilde{H}^{t, t^{\prime}}(\sigma)$, it is often easiest first to extend $u$ to an element $\tilde{u} \in H_{\mathrm{loc}}^{t,-\infty}\left(\mathbf{R}^{n+1}\right)$ and then to find an operator $\phi\left(z, D^{\prime}\right) \in T^{0}\left(\mathbf{R}^{n+1}\right)$, elliptic at $\sigma$, such that $\phi \tilde{u} \in$ $H_{\text {loc }}^{t, t^{\prime}}\left(\mathbf{R}^{n+1}\right)$.

First we state a microlocal algebra result for interior points.

Lemma 2.2 (Rauch [11]). For any $\lambda \in T^{*} \stackrel{\circ}{\Omega} \backslash 0$, if $u \in H_{\mathrm{loc}}^{s_{1}}(\stackrel{\circ}{\Omega}) \cap H^{r}(\lambda)$ and $v \in$ $H_{\mathrm{loc}}^{s_{2}}(\stackrel{\circ}{\Omega}) \cap H^{r}(\lambda), s_{i}>(n+1) / 2$, then $u v \in H^{r}(\lambda)$ provided $r<s_{1}+s_{2}-(n+1) / 2$.

Lemma 2.3 (Sablé-Tougeron [13]). The spaces $H_{\mathrm{loc}}^{t, t^{\prime}}(\Omega)$ are algebras stable under composition with smooth $f(z, u)$ if $t>\frac{1}{2}, t+t^{\prime}>(n+1) / 2, t+2 t^{\prime}>\frac{1}{2}$.

This lemma and Peetre's theorem yield by induction the simple

Corollary 2.4 [7, Proposition 2.15]. If $u \in H_{\mathrm{loc}}^{s}(\Omega), s>(n+1) / 2$, satisfies $\square u=f(u)$ for $f \in C^{\infty}$, then $u \in H_{\mathrm{loc}}^{2 s-\alpha+2,-s+\alpha-2}(\Omega)$ for all $\alpha>\frac{1}{2}$.

Thus $\widetilde{W F}_{t, t^{\prime}} u$ is defined for such solutions as long as $t<2 s+\frac{3}{2}$. The next result is an analogue of Rauch's lemma at the boundary.

Lemma 2.5 ([14, Lemma 1.10]; also [13]). Suppose $s^{\prime}, t^{\prime}$ satisfy $(n+2) / 2$ $<s \leq s^{\prime}<2 s-(n+1) / 2-\alpha, 0 \leq t^{\prime} \leq \alpha$, where $\alpha \in\left(\frac{1}{2}, s-(n+1) / 2\right)$. Then if $u, v \in H_{\mathrm{loc}}^{2 s-\alpha,-s+\alpha}(\Omega) \cap \widetilde{H}^{s^{\prime}, t^{\prime}}(\sigma)$ for some $\sigma \in T^{*} b \Omega \backslash 0$,

$$
u v \in H_{\mathrm{loc}}^{2 s-\alpha,-s+\alpha}(\Omega) \cap \widetilde{H}^{s^{\prime}, t^{\prime}}(\sigma) .
$$

We proceed now to state the linear and nonlinear propagation theorems that will be used in the analysis of the remainder $R \beta\left(u^{2}-v^{2}\right)$. First recall that the elliptic, hyperbolic, and glancing regions $(E, H$, and $G)$ of $T^{*} b \Omega \backslash 0$, defined with respect to $\square$, consist respectively of points $(t, y, \tau, \eta)$ such that $\tau^{2}<|\eta|^{2}, \tau^{2}>|\eta|^{2}, \tau^{2}=|\eta|^{2}$. The bicharacteristics of $\tau^{2}-|\eta|^{2}$ in $G$ are called gliding rays. Given $\sigma \in H$ we shall denote by $\gamma_{-}(\sigma)$ (resp. $\gamma_{+}(\sigma)$ ) the open half-ray (a null bicharacteristic of $\square$ ) in $T^{*} \Omega$, with endpoint over $\sigma$, on which $d x / d t<0$ (resp. $d x / d t>0$ ). Even for the operator $\square$ on this simple domain, it is convenient to describe propagation in terms of generalized bicharacteristics.

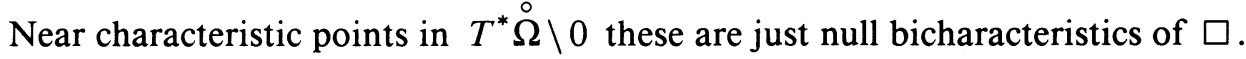
For $\sigma \in H \cup G$ let us denote by $\Gamma(t)$ the generalized bicharacteristic through $\sigma$. Then if $\sigma \in G, \Gamma(t)$ is the gliding ray through $\sigma$. If $\sigma \in H, \Gamma(t)$ coincides near $\sigma$ with the continuous curve obtained by taking $\gamma_{-}(\sigma) \cup\{\sigma\} \cup \gamma_{+}(\sigma)$ and identifying the points over $b \Omega$. (A precisely stated, general definition is given in [9].)

The following linear propagation theorem is a special case of Theorem 1.3 of [14]. 
Theorem 2.6. Suppose $u \in D^{\prime}(\Omega)$ (the space of extendible distributions) satisfies $\square u=f$ in $\stackrel{\circ}{\Omega}$, where for some $s^{\prime} \geq 1, t^{\prime} \geq-1, f \in H_{\mathrm{loc}}^{s^{\prime},-\infty}(\Omega)$ and $u \in$ $H^{s^{\prime}+t^{\prime}+3 / 2}\{(t, x, y, 0, \xi, 0): x>0, \xi \neq 0,(t, x, y)$ near $b \Omega\}$. Then every point $\gamma_{0} \in \widetilde{W F}_{s^{\prime}+2, t^{\prime}-1} u \backslash\left(\widetilde{W F}_{s^{\prime}, t^{\prime}} f \cup W F_{s^{\prime}+t^{\prime}+1 / 2}\left(\left.u\right|_{b \Omega}\right)\right)$ is either a characteristic point of $\square$ in $T^{*} \stackrel{\circ}{\Omega}$ or else contained in $H \cup G$. If $p, q$ satisfy $0 \leq p \leq s^{\prime}+2$, $p+q=s^{\prime}+t^{\prime}+1$ and $\gamma_{0} \in \widetilde{W F}_{p, q} u \backslash\left(\widetilde{W F}_{s^{\prime}, t^{\prime}} f \cup W F_{s^{\prime}+t^{\prime}+1}\left(\left.u\right|_{b \Omega}\right)\right)$, then an open interval $(-N, N) \ni t \rightarrow \Gamma(t)$ with $\Gamma(0)=\gamma_{0}$ on a generalized bicharacteristic is contained in $\widetilde{W F}_{p, q} u$.

An iterative argument combining Lemma 2.5 with Theorem 2.6 gives the following nonlinear $2 s$ theorem, a special case of Theorem 1.11 of [14].

Theorem 2.7. Let $f(z, u) \in C^{\infty}$, but if $\operatorname{Im} u \neq 0$ assume $f$ is analytic in $u$. Suppose that $u \in H_{\mathrm{loc}}^{s}(\Omega), s>(n+2) / 2$, satisfies $\square u=f(z, u)$ in $\stackrel{\circ}{\Omega}$. Then for $p, q$ such that $s \leq p, 0 \leq q \leq 1 / 2, p+q<2 s-(n+1) / 2+1$, every point $\gamma_{0} \in \widetilde{W F}_{p, q} u \backslash W F_{p+q}\left(\left.u\right|_{b \Omega}\right)$ is either a characteristic point of $\square$ in $T^{*} \Omega$ or else contained in $H \cup G$. Moreover, an open interval $(-N, N) \ni t \rightarrow \Gamma(t)$ with $\Gamma(0)=\gamma_{0}$ on a generalized bicharacteristic is contained in $\widetilde{W F}, q$.

In the estimates to follow the next fact is often used (see [12]). Let $\beta=$ $\left(\tau_{0}, \xi_{0}, \eta_{0}\right)$ be another symbol for the dual variables.

(2.8) If $h(\zeta)=\int G(\zeta, \beta) f(\zeta-\beta) g(\beta) d \beta$ where $f, g \in L^{2}$ and $G$ can be written as a sum of finitely many functions $G_{i}$ with $\sup _{\zeta} \int\left|G_{i}\right|^{2} d \beta<\infty$ or $\sup _{\beta} \int\left|G_{i}\right|^{2} d \zeta<\infty$, then one has $\|h\|_{2}<C\|f\|_{2}\|g\|_{2}$.

\section{CROSSING AT THE BOUNDARY}

Most of this section is devoted to part (a) of Theorem 1.9. At the end we shall explain the changes needed to prove part (b).

We will use the notation and reductions described in the introduction. In a single proof the letter $C$ may denote several different constants, and the same applies to $\varepsilon$ and $M$. Note also that in the rest of the paper we will represent $(\tau, \eta)$ by $\mu$ instead of $\zeta^{\prime}$.

\subsection{The singularities of $R \beta v^{2}$.}

Proposition 3.1. Let $w$ be as in (1.4)(a), define $v$ as in (1.14), and fix $\varepsilon>0$ arbitrarily small. Then if $\rho$ in (1.3) is taken close enough to $1, R \beta v^{2}$ has singularities of strength $2 s-n / 2+2+\varepsilon$ throughout $\Lambda_{1}$; that is, $R \beta v^{2} \notin$ $H^{2 s-n / 2+2+\varepsilon}(\lambda)$ for all $\lambda \in \Lambda_{1}$.

The proof consists of several lemmas. As explained in the introduction, we need only consider $C\left(\left.\left(E \beta v^{2}\right)\right|_{b \Omega}\right)$. In fact, it will be clear from the following analysis that it is really enough to exhibit singularities as above in

$$
C\left(\left.\left(E \beta v_{a 1} v_{a 2}\right)\right|_{b \Omega}\right)-C\left(\left.\left(E \beta v_{a 1} v_{b 2}\right)\right|_{b \Omega}\right) \quad(\text { recall }(1.18)-(1.20)) \text {. }
$$


The first lemma shows that for the purpose of detecting singularities of $C\left(\left.\left(E \beta v_{a 1} v_{a 2}\right)\right|_{b \Omega}\right)$ on the outgoing ray over any fixed $(0, \bar{\mu}) \in T^{*} b \Omega \backslash 0$ with $\bar{\mu} \in \stackrel{\circ}{C}_{1}, E$ can be replaced by a simple multiplier. Without loss of generality we take $\bar{\mu}=(1,0)$. A similar result holds, of course, for the second term in (3.2).

Lemma 3.3. Let $H(\tau, \xi, \eta)$ be the characteristic function of a small conic neighborhood of $(2, \sqrt{2}, 0)=\left(1, \omega_{1}\right)+\left(1, \omega_{2}\right) \in B^{\omega_{1}, \omega_{2}}$, and set

$$
\widehat{U}=\left(H(\zeta) /\left(\tau^{2}-|\xi, \eta|^{2}\right)\right) \widehat{\beta v_{a 1} v_{a 2}} .
$$

Then $W F\left[C\left(\left.\left(E \beta v_{a 1} v_{a 2}\right)\right|_{b \Omega}\right)-C\left(\left.U\right|_{b \Omega}\right)\right] \cap \gamma_{+}(0, \bar{\mu})=\varnothing$.

Proof. Let $V=E \beta v_{a 1} v_{a 2}-C\left(\left.\left(E \beta v_{a 1} v_{a 2}\right)\right|_{b \Omega}\right)$ and set $\widetilde{V}=U-C\left(\left.U\right|_{b \Omega}\right)$. Then $V-\widetilde{V}$ satisfies, in $\Omega$,

$\square(V-\widetilde{V})=(1-H(D)) \beta v_{a 1} v_{a 2},\left.\quad(V-\widetilde{V})\right|_{b \Omega}=0, \quad V-\widetilde{V} \in C^{\infty} \quad$ in $t<-\delta$.

Since $\widehat{\beta v_{a 1} v_{a 2}}$ is rapidly decreasing outside $B^{\omega_{1}, \omega_{2}}$ (and because of the location of supp $H$ ), we have $W F(1-H(D)) \beta v_{a 1} v_{a 2} \cap\left(\gamma_{-}(0, \bar{\mu}) \cup \gamma_{+}(0, \bar{\mu})\right)=\varnothing$ and $(1-H(D)) \beta v_{a 1} v_{a 2} \in \widetilde{H}^{\infty}(0, \bar{\mu})$. Hence, by classical results on reflection of regularity (or Theorem 2.6), (3.4) implies $W F(V-\widetilde{V}) \cap \gamma_{+}(0, \bar{\mu})=\varnothing$, which in turn implies the desired result.

So now we concentrate on measuring the singularities of $C\left(\left.U\right|_{b \Omega}\right)$. The first step is to get a lower bound for $\widehat{\beta v_{a 1} v_{a 2}}$ on a subconic neighborhood of each point in $\stackrel{\circ}{B}^{\omega_{1}, \omega_{2}}$. We will do this for $\bar{\zeta}=(\sqrt{2 / 3}, \sqrt{1 / 3}, 0)$, the argument being the same for other points on the wedge.

Lemma 3.5. There exist constants $\varepsilon, M$ such that on $S=\{\zeta=(\tau, \xi, \eta):|\zeta| \geq$ $\left.M,|\zeta-\bar{\zeta}| \zeta|| \leq \varepsilon|\zeta|^{1 / \rho}\right\}$ we have $\widehat{\beta v_{a 1} v_{a 2}}(\zeta) \geq C\langle\zeta\rangle^{-(2 s+1+O(\rho-1))}$.

Proof. It suffices to show that the conclusion holds for $\zeta \in S^{\prime}=\{(\tau, \xi, \eta)$ : $\left.|(\xi, \eta)-(1,0)| \xi, \eta|| \leq \varepsilon|\xi, \eta|^{1 / \rho},|\tau-\sqrt{2}| \xi, \eta|| \leq \varepsilon|\xi, \eta|^{1 / \rho},|\xi, \eta| \geq M\right\}$. We must estimate

$$
\int \widehat{\beta}\left(\tau-\left|\xi-\xi_{0}, \eta-\eta_{0}\right|-\left|\xi_{0}, \eta_{0}\right|\right) \hat{f}_{1}\left(\xi-\xi_{0}, \eta-\eta_{0}\right) \hat{f}_{2}\left(\xi_{0}, \eta_{0}\right) d \xi_{0} d \eta_{0} .
$$

Fix a $(\tau, \xi, \eta) \in S^{\prime}$. So $\tau=\sqrt{2}|\xi, \eta|+\delta|\xi, \eta|^{1 / \rho}$ for some $|\delta| \leq \varepsilon$. Recalling that $\widehat{\beta} \geq 0, \widehat{\beta}(0)>0$ we choose $\delta^{\prime}, C$ such that $\widehat{\beta}(\sigma) \geq C$ for $|\sigma| \leq \delta^{\prime}$. Now define

(3.7) $A=\bigcup_{\left|\varepsilon^{\prime}\right| \leq \delta^{\prime}}\left\{\left(\xi_{0}, \eta_{0}\right): \sqrt{2}|\xi, \eta|+\delta|\xi, \eta|^{1 / \rho}+\varepsilon^{\prime}=\left|\xi-\xi_{0}, \eta-\eta_{0}\right|+\left|\xi_{0}, \eta_{0}\right|\right\}$,

a union of confocal ellipsoids with foci at $(\xi, \eta), 0$. Noting that $|\xi, \eta| \omega_{2} / \sqrt{2}$ $\left(\omega_{2}=(1 / \sqrt{2},-1 / \sqrt{2}, 0)\right)$ lies on the ellipsoid $\sqrt{2}|\xi, \eta|=|| \xi, \eta \mid(1,0)-$ $\left(\xi_{0}, \eta_{0}\right)|+| \xi_{0}, \eta_{0} \mid$, we define

$$
B=\left\{\left(\xi_{0}, \eta_{0}\right):\left|\left(\xi_{0}, \eta_{0}\right)-\right| \xi, \eta\left|\omega_{2} / \sqrt{2}\right| \leq \varepsilon|\xi, \eta|^{1 / \rho}\right\} .
$$


For $\left(\xi_{0}, \eta_{0}\right) \in B$ and $M$ large, it is easy to check that $\left\langle\xi_{0}, \eta_{0}\right\rangle \sim\langle\xi, \eta\rangle$, $\left\langle\xi-\xi_{0}, \eta-\eta_{0}\right\rangle \sim\langle\xi, \eta\rangle,\left|\left(\xi_{0}, \eta_{0}\right)-\right| \xi_{0}, \eta_{0}\left|\omega_{2}\right| \leq C \varepsilon\left|\xi_{0}, \eta_{0}\right|^{1 / \rho}$, and

$$
\left|\left(\xi-\xi_{0}, \eta-\eta_{0}\right)-\right| \xi-\xi_{0}, \eta-\eta_{0}\left|\omega_{1}\right| \leq C \varepsilon\left|\xi-\xi_{0}, \eta-\eta_{0}\right|^{1 / \rho}
$$

So, using (1.3)(c) we get

$$
\begin{aligned}
& \widehat{\beta v_{a 1} v_{a 2}} \geq C \int_{A \cap B} \hat{f}_{1}\left(\xi-\xi_{0}, \eta-\eta_{0}\right) \hat{f}_{2}\left(\xi_{0}, \eta_{0}\right) d \xi_{0} d \eta_{0} \\
& \geq C \int_{A \cap B}\langle\xi, \eta\rangle^{-2(s+n / 2+0(\rho-1))} d \xi_{0} d \eta_{0} .
\end{aligned}
$$

Since $\operatorname{vol}(A \cap B) \geq C\left(|\xi, \eta|^{1 / \rho}\right)^{n-1}$ for a $C$ independent of $(\tau, \xi, \eta) \in S^{\prime}$, the result follows.

Next we derive a lower bound for $\widehat{\left.U\right|_{b \Omega}}$ on a subconic neighborhood of $\bar{\mu}=(1,0)$. Note that $\left.\operatorname{sing} \operatorname{supp} U\right|_{b \Omega}=\{0\}$.

Lemma 3.10. There exist constants $\varepsilon, M$ such that for $\mu=(\tau, \eta)$ satisfying $|\mu| \geq M,|\mu-(1,0)| \mu \| \leq \varepsilon|\mu|^{1 / \rho}$ we have $\widehat{\left.U\right|_{b \Omega}} \geq C\langle\mu\rangle^{-(2 s+2+O(\rho-1))}$.

Proof. For $\zeta \in S$ (as in Lemma 3.5), since $H$ is supported near $\bar{\zeta}$ we have

$$
\widehat{U}=\frac{H(\zeta)}{\tau^{2}-|\xi, \eta|^{2}} \widehat{\beta v_{a 1} v_{a 2}} \geq C\langle\zeta\rangle^{-(2 s+3+O(\rho-1))} .
$$

We must estimate

$$
\widehat{\left.U\right|_{b \Omega}}=\int \frac{H(\zeta)}{\tau^{2}-|\xi, \eta|^{2}} \widehat{\beta v_{a 1} v_{a 2}} d \xi
$$

Now $S$ contains a set of the form $S^{\prime \prime}=\left\{(\tau, \xi, \eta):|\mu-(1,0)| \mu|| \leq \varepsilon|\mu|^{1 / \rho}\right.$, $\left.|\xi-| \mu|/ \sqrt{2}| \leq \varepsilon|\mu|^{1 / \rho},|\mu| \geq M\right\}$. Setting $a_{ \pm}(\mu)=|\mu| / \sqrt{2} \pm \varepsilon|\mu|^{1 / \rho}$ and $t=2 s+2+O(\rho-1)$, we obtain that for $M$ large and $\mu$ as in the definition of $S^{\prime \prime}$

$$
\begin{aligned}
\widehat{\left.U\right|_{b \Omega}} & \geq C \int_{a_{-}(\mu)}^{a_{+}(\mu)}(\langle\mu\rangle+\xi)^{-(t+1)} d \xi \\
& \geq C\langle\mu\rangle^{-(t+1-1 / \rho)}\left[\langle\mu\rangle^{t+1-1 / \rho} \cdot \frac{\langle\mu\rangle^{t-1}\left(a_{+}-a_{-}\right)}{\langle\mu\rangle^{2 t}}\right] \\
& \geq C\langle\mu\rangle^{-(t+1-1 / \rho)} .
\end{aligned}
$$

The next lemma shows that the first term in (3.2) has singularities of the desired strength on $\Lambda_{1}$.

Lemma 3.14. Fix $\varepsilon>0$. If $\rho$ in (1.3) is chosen close enough to 1 , then for all $\lambda \in \Lambda_{1}, C\left(\left.\left(E \beta v_{a 1} v_{a 2}\right)\right|_{b \Omega}\right) \notin H^{2 s-n / 2+2+\varepsilon}(\lambda)$.

Proof. Without loss of generality we shall look near $\gamma_{+}(0, \bar{\mu}), \bar{\mu}=(1,0)$. So let $\left(t_{0}, x_{0}, y_{0}, 1,-1,0\right) \in \gamma_{+}(0, \bar{\mu})$. Choose $\alpha(x) \in C_{0}^{\infty}(\mathbf{R})$ with support 
concentrated near $x_{0}$ such that $|\widehat{\alpha}(0)|>0$, and denote by $p(\tau, \xi, \eta)$ the characteristic function of a small conic neighborhood of $(1,-1,0)$. With $U$ as in (3.11) set $h(\tau, \eta)=\widehat{\left.U\right|_{b \Omega}}$ and note that $C\left(\left.U\right|_{b \Omega}\right)$ is equal $\left(\bmod C^{\infty}\right)$ to

$$
C^{\prime}\left(\left.U\right|_{b \Omega}\right)=(2 \pi)^{-n} \int \exp \left(i t \tau-i x \sqrt{\tau^{2}-|\eta|^{2}}+i y \eta\right) h(\tau, \eta) d \tau d \eta .
$$

We claim that if $\rho$ is chosen close enough to 1 ,

$$
\langle\tau, \xi, \eta\rangle^{2 s-n / 2+2+\varepsilon} p(\tau, \xi, \eta) \widehat{\alpha C^{\prime}\left(\left.U\right|_{b \Omega}\right)} \notin L^{2}\left(R^{n+1}\right) .
$$

To see this, observe that by $(3.15), \widehat{\alpha C^{\prime}\left(\left.U\right|_{b \Omega}\right)}=\widehat{\alpha}\left(\xi+\sqrt{\tau^{2}-|\eta|^{2}}\right) h(\tau, \eta)$. Hence, if (3.16) were not true, that would imply

$$
\langle\tau, \eta\rangle^{2 s-n / 2+2+\varepsilon} h(\tau, \eta) \in L^{2}\left(R^{n}\right),
$$

but (3.17) does not hold if $\rho$ is close enough to 1 , by Lemma 3.10. Since $W F C^{\prime}\left(\left.U\right|_{b \Omega}\right)$ is concentrated near $\gamma_{+}(0, \bar{\mu})$, the desired conclusion now follows from (3.16) and Lemma 3.3.

Lemma 3.18. Fix $\varepsilon>0$. If $\rho$ in (1.3) is chosen close enough to 1 , then for all $\lambda \in \Lambda_{1}, C\left(\left.\left(E \beta v_{a 1} v_{a 2}\right)\right|_{b \Omega}\right)-C\left(\left.\left(E \beta v_{a 1} v_{b 2}\right)\right|_{b \Omega}\right) \notin H^{2 s-n / 2+2+\varepsilon}(\lambda)$.

Proof. Again we focus on $\gamma_{+}(0, \bar{\mu}), \bar{\mu}=(1,0)$. Note that $\pi_{2} W F \beta v_{a 1} v_{b 2}=$ $B^{\omega_{1}, \omega_{2}^{*}}$, where $\omega_{2}^{*}=(-1 / \sqrt{2},-1 / \sqrt{2}, 0)$, and that $(1,0,0) \in B^{\omega_{1}, \omega_{2}^{*}}$ projects to $\bar{\mu}$. Let $\widetilde{H}(\zeta)$ be the characteristic function of a small conic neighborhood of $(1,0,0)$ and set

$$
\widehat{V}=\frac{\widetilde{H}(\zeta)}{\tau^{2}-|\xi, \eta|^{2}} \widehat{\beta v_{a 1} v_{b 2}} .
$$

In view of Lemma 3.3 and the proof of Lemma 3.14, it will suffice to show that there exist constants $\varepsilon, M$ such that for $\mu=(\tau, \eta)$ satisfying $|\mu| \geq M$, $|\mu-(1,0)| \mu|| \leq \varepsilon|\mu|^{1 / \rho}$ we have

$$
\widehat{\left.U\right|_{b \Omega}}-\widehat{\left.V\right|_{b \Omega}} \geq C\langle\mu\rangle^{-(2 s+2+O(\rho-1))} .
$$

We must compare

$$
\begin{aligned}
\widehat{\left.V\right|_{b \Omega}}=\int \frac{\tilde{H}(\zeta)}{\tau^{2}-|\xi, \eta|^{2}} & \widehat{\beta}\left(\tau-\left|\xi-\xi_{0}, \eta-\eta_{0}\right|-\left|\xi_{0}, \eta_{0}\right|\right) \\
& \cdot \hat{f}_{1}\left(\xi-\xi_{0}, \eta-\eta_{0}\right) \hat{f}_{2}\left(-\xi_{0}, \eta_{0}\right) d \xi_{0} d \eta_{0} d \xi
\end{aligned}
$$

with (3.12). After the change of variables $\xi^{\prime}=\xi-2 \xi_{0}, \tilde{\xi}=-\xi_{0}$, (3.21) becomes (relabeling $\xi^{\prime}=\xi, \widetilde{\xi}=\xi_{0}$ )

$$
\begin{aligned}
\int \frac{\widetilde{H}\left(\tau, \xi-2 \xi_{0}, \eta\right)}{\tau^{2}-\left|\xi-2 \xi_{0}, \eta\right|^{2}} \widehat{\beta}\left(\tau-\left|\xi-\xi_{0}, \eta-\eta_{0}\right|-\left|\xi_{0}, \eta_{0}\right|\right) \\
\cdot \hat{f}_{1}\left(\xi-\xi_{0}, \eta-\eta_{0}\right) \hat{f}_{2}\left(\xi_{0}, \eta_{0}\right) d \xi_{0} d \eta_{0} d \xi
\end{aligned}
$$


Let $F\left(\tau, \xi, \eta, \xi_{0}, \eta_{0}\right)$ denote the integrand in (3.22), and note that

$$
\int F d \xi_{0} d \eta_{0} \leq C \chi(\mu) \widehat{\beta v_{a 1} v_{a 2}},
$$

where $\chi$ is the characteristic function of a small conic neighborhood of $\bar{\mu}$. Since $\widehat{\beta v_{a 1} v_{a 2}}$ is rapidly decreasing outside $B^{\omega_{1}, \omega_{2}}$, it follows from (3.23) that, with $\Gamma$ a small conic neighborhood of $(\sqrt{2 / 3}, \sqrt{1 / 3}, 0) \in B^{\omega_{1}, \omega_{2}}$ and $D(\tau, \eta)=\left\{\left(\xi_{0}, \eta_{0}, \xi\right):(\tau, \xi, \eta) \in \Gamma\right\}$,

$$
\int F d \xi_{0} d \eta_{0} d \xi=\int_{D(\tau, \eta)} F d \xi_{0} d \eta_{0} d \xi+O\left(\langle\tau, \eta\rangle^{-\infty}\right) .
$$

Thus, it is enough to compare (3.12) with the integral over $D(\tau, \eta)$ in (3.24). Now given any $\delta>0$, if $\Gamma$ and supp $\widetilde{H}$ are small enough (as we are free to arrange), then for $(\tau, \xi, \eta) \in \Gamma,\left(\tau, \xi-2 \xi_{0}, \eta\right) \in \operatorname{supp} \widetilde{H},\langle\tau, \eta\rangle$ large, we have

$$
\left(\tau^{2}-\left|\xi-2 \xi_{0}, \eta\right|^{2}\right)^{-1} \leq\left(\frac{1}{2}+\delta\right)\left(\tau^{2}-|\xi, \eta|^{2}\right)^{-1}
$$

With Lemma 3.10 this implies the estimate (3.20).

Remark 3.26. The proof shows that the basic reason why the first term dominates the second in (3.20) is simply this: the ray on $B^{\omega_{1}, \omega_{2}}$ which projects to $\{t \bar{\mu}: t>0\}$ under $(\tau, \xi, \eta) \rightarrow(\tau, \eta)$ lies closer to $\tau^{2}=|\xi, \eta|^{2}$ than the ray on $B^{\omega_{1}, \omega_{2}^{*}}$ with that projection. Of course, analogous statements hold with respect to other choices of $\bar{\mu} \in \stackrel{\circ}{C}_{1}$. This is perhaps the easiest way to see why incoming-incoming or outgoing-outgoing interactions dominate incomingoutgoing interactions (recall (1.21)). Note that if the incoming rays are quite close to being gliding rays, the singularities due to incoming-outgoing interactions are nearly as strong as those due to incoming-incoming interactions.

Since $C\left(\left.\left(E \beta v_{a 1} v_{a 2}\right)\right|_{b \Omega}\right)-C\left(\left.\left(E \beta v_{a 2} v_{b 1}\right)\right|_{b \Omega}\right)$ can be analyzed in the same way, Lemma 3.18 implies that $v_{a}^{2}-v_{a} v_{b}$ contributes singularities of strength $2 s-n / 2+2+\varepsilon$ to $R \beta v^{2}$ on $\Lambda_{1}$. Applying the same arguments to $v_{b}^{2}-v_{a} v_{b}$ shows that it contributes singularities of the same strength and sign. So this concludes the proof of Proposition 3.1.

3.2. The remainder $R \beta\left(u^{2}-v^{2}\right)$. Proposition 3.1 and the following proposition together imply Theorem 1.9(a).

Proposition 3.27. In the case of Theorem 1.9(a), $R \beta\left(u^{2}-v^{2}\right) \in H^{t}(\lambda)$ for all $t<2 s-n / 2+\frac{5}{2}$, for all $\lambda \in \Lambda_{1}$.

Recall from the introduction that $R \beta\left(u^{2}-v^{2}\right)=2 R \beta\left(v R \beta u^{2}\right)+R \beta\left(R \beta u^{2}\right)^{2}$. The second term can be handled using the results of $\S 2$ and the following lemma. Here we let $\pi\left(1, \omega_{1}\right)=\mu_{1}$ and $\pi\left(1, \omega_{2}\right)=\mu_{2}(\pi(\tau, \xi, \eta)=(\tau, \eta))$.

Lemma 3.28. Away from $z=0 \in \mathbf{R}^{n+1}, W F v^{2}=W F v$. If $\bar{\mu} \in \mathbf{R}^{n} \backslash 0$ lies outside a conic neighborhood of $\left\{\mu_{1}, \mu_{2}\right\}$, then for $\sigma=(0, \bar{\mu}) \in T^{*} b \Omega \backslash 0$, $v^{2} \in \widetilde{H}^{2 s-n / 2+\varepsilon}(\sigma)$ for all $\varepsilon<\frac{1}{2}$. 
Note that Lemma 2.5 gives regularity only up to order $2 s-(n+1) / 2$ for such $\sigma$. Actually, for $\sigma=(0, \bar{\mu})$ with $\bar{\mu} \notin C_{1}$, it is easy to see that $v^{2} \in \widetilde{H}^{t}(\sigma)$ for all $t$, but we will not need this.

Proof. The first statement is clear since interaction occurs only at 0 . The second follows from [1, Lemma 5], but we give here a slightly simpler proof by a method that will also be useful elsewhere.

Consider first $v_{a 1} v_{a 2}$. For any $\alpha(t) \in C_{0}^{\infty}$,

$$
\widehat{\alpha v_{a 1}}=\widehat{\alpha}(\tau-|\xi, \eta|) \hat{f}_{1}(\xi, \eta)=\gamma(\tau-|\xi, \eta|) \hat{h}_{1}(\tau, \xi, \eta)
$$

for some $\gamma \in \mathscr{S}, h_{1} \in H^{s}\left(R^{n+1}\right)$. Similarly, $\widehat{\alpha v_{a 2}}=\gamma(\tau-|\xi, \eta|) \hat{h}_{2}$ for some $h_{2} \in H^{s}\left(R^{n+1}\right)$. Let $\chi_{1}, \chi_{2}$ be characteristic functions of small conic neighborhoods of $\left(1, \omega_{1}\right),\left(1, \omega_{2}\right)$, respectively, and let $\chi(\mu)$ be the characteristic function of a small conic neighborhood of $\bar{\mu}$. It is clearly enough to examine $\left(\chi_{1}(D) \alpha v_{a 1}\right)\left(\chi_{2}(D) \alpha v_{a 2}\right)$. For this we shall apply (2.8) to

$$
G(\zeta, \beta)=\frac{\chi(\mu)\langle\zeta\rangle^{2 s-n / 2+\varepsilon} \gamma\left(\tau-\tau_{0}-\left|\xi-\xi_{0}, \eta-\eta_{0}\right|\right) \gamma\left(\tau_{0}-\left|\xi_{0}, \eta_{0}\right|\right) \chi_{1}(\zeta-\beta) \chi_{2}(\beta)}{\langle\zeta-\beta\rangle^{s}\langle\beta\rangle^{s}}
$$

On $\operatorname{supp} G|\zeta-\beta| \geq C|\zeta|$ and $|\beta| \geq C|\zeta|$ so $|G| \leq F=$

$$
\frac{C\left|\gamma\left(\tau-\tau_{0}-\left|\xi-\xi_{0}, \eta-\eta_{0}\right|\right) \gamma\left(\tau_{0}-\left|\xi_{0}, \eta_{0}\right|\right)\right| \chi_{1}(\zeta-\beta) \chi_{2}(\beta)}{\langle\beta\rangle^{n / 2-\varepsilon}} .
$$

Writing $\eta_{0}=\left(\eta_{01}, \eta_{0}^{\prime}\right)$, we define the map $T:\left(\tau_{0}, \eta_{01}\right) \rightarrow\left(\tilde{\tau}, \tilde{\eta}_{1}\right)$ by

$$
\tilde{\tau}=\tau_{0}-\left|\xi_{0}, \eta_{0}\right|, \quad \tilde{\eta}_{1}=\tau-\tau_{0}-\left|\xi-\xi_{0}, \eta-\eta_{0}\right| \text {. }
$$

Note that

$$
\operatorname{det}(d T)=\frac{\eta_{1}-\eta_{01}}{\left|\xi-\xi_{0}, \eta-\eta_{0}\right|}-\frac{\eta_{01}}{\left|\xi_{0}, \eta_{0}\right|} \geq C>0 \quad \text { on supp } G
$$

Hence,

$$
\begin{aligned}
\int F^{2} d \beta & =\int\left(F \circ T^{-1}\right)^{2}\left|\operatorname{det} d T^{-1}\right| d \tilde{\tau} d \tilde{\eta}_{1} d \xi_{0} d \eta_{0}^{\prime} \\
& \leq C \int \frac{\left|\gamma^{2}(\tilde{\tau}) \gamma^{2}\left(\tilde{\eta}_{1}\right)\right|}{\left\langle\xi_{0}, \eta_{0}^{\prime}\right\rangle^{n-2 \varepsilon}} d \tilde{\tau} d \tilde{\eta}_{1} d \xi_{0} d \eta_{0}^{\prime}<\infty
\end{aligned}
$$

if $\varepsilon<\frac{1}{2}$. By (2.8) this finishes the case $v_{a 1} v_{a 2}$. The case $v_{a 1} v_{b 2}$ can be handled by the same change of variables. The remaining terms constituting $v^{2}$ either satisfy the conditions of the lemma trivially or can be handled as above.

Lemma 3.33. In the case of Theorem 1.9(a), $R \beta\left(R \beta u^{2}\right)^{2} \in H^{t}(\lambda)$ for all $t<$ $2 s-n / 2+\frac{5}{2}$, for all $\lambda \in \Lambda_{1}$.

Proof. For any $\bar{\mu} \in \stackrel{\circ}{C}_{1}$ let $\sigma=(0, \bar{\mu}) \in T^{*} b \Omega \backslash 0$, and for $t \in \mathbf{R}$ denote by $\mathscr{H}^{t}(\sigma)$ the space $H^{t}\left(\gamma_{-}(\sigma)\right) \cap \widetilde{H}^{t}(\sigma) \cap H^{t}\left(\gamma_{+}(\sigma)\right)$. First we show

$$
u^{2} \in \mathscr{H}^{t}(\sigma) \text { for } t<2 s-n / 2+\frac{1}{2} \text {. }
$$


By Theorem 2.7, $u \in \mathscr{H}^{t}(\sigma)$ for $t<2 s-n / 2+\frac{1}{2}$. Rauch's lemma then gives $u^{2} \in H^{t}\left(\gamma_{-}(\sigma) \cup \gamma_{+}(\sigma)\right)$ for $t<2 s-(n+1) / 2$, and Lemma 2.5 implies $u^{2} \in \widetilde{H}^{s^{\prime}, t^{\prime}}(\sigma)$ for $s^{\prime}<2 s-(n+1) / 2-\alpha, t^{\prime}=\alpha$ for all $\alpha>\frac{1}{2}$. Applying the linear propagation theorem, Theorem 2.6, we obtain $R \beta u^{2} \in \mathscr{H}^{t}(\sigma)$ for $t<2 s-n / 2+\frac{1}{2}$. Another application of Rauch's lemma and Lemma 2.5 (using the fact that $\left.R \beta u^{2} \in H_{\mathrm{loc}}^{2(s+1)-\alpha,-(s+1)+\alpha}\left(\Omega_{T}\right)\right)$ shows that $\left(R \beta u^{2}\right)^{2} \in \mathscr{H}^{t}(\sigma)$ for $t<2 s-n / 2+\frac{1}{2}$. Now to prove (3.34) we write $u^{2}=v^{2}+2 v R \beta u^{2}+\left(R \beta u^{2}\right)^{2}$, and note that by Lemma 3.28 it remains only to consider $v R \beta u^{2}$. We have $v \in H_{\mathrm{loc}}^{s+N},-N(\Omega) \cap \mathscr{P}^{N}(\sigma)$ for all $N$ and $R \beta u^{2} \in H_{\mathrm{loc}}^{2(s+1)-\alpha,-(s+1)+\alpha}\left(\Omega_{T}\right) \cap \mathscr{H}^{t}(\sigma)$ for $t<2 s-n / 2+\frac{1}{2}$. Rauch's lemma yields $v R \beta u^{2} \in H^{t}\left(\gamma_{-}(\sigma) \cup \gamma_{+}(\sigma)\right)$ for $t<2 s-n / 2+\frac{1}{2}$, and (the proof of) Lemma 2.5 gives $v R \beta u^{2} \in \widetilde{H}^{t}(\sigma)$ for such $t$. This establishes (3.34).

To finish, observe that (3.34) and Theorem 2.6 imply $R \beta u^{2} \in \mathscr{H}^{t}(\sigma)$ for $t<2 s-n / 2+\frac{3}{2}$. The algebra lemmas give $\left(R \beta u^{2}\right)^{2} \in \mathscr{H}^{t}(\sigma)$ for such $t$, and a final application of Theorem 2.6 concludes the proof.

Remark 3.35. Suppose $\bar{\mu} \in \mathbf{R}^{n} \backslash 0$ lies outside a conic neighborhood of $\left\{\mu_{1}, \mu_{2}\right\}$. If Theorems 2.6 and 2.7 are used fully (i.e., not just the parts concerned with rays over hyperbolic points), then the argument used to prove (3.34) shows that for $\sigma=(t, y, \bar{\mu}) \in T^{*} b \Omega_{T} \backslash 0, u^{2} \in \widetilde{H}^{2 s-n / 2+\varepsilon}(\sigma)$ for all $\varepsilon<\frac{1}{2}$. It also shows that if $\lambda=(z, \zeta) \in T^{*} \stackrel{\circ}{\Omega}_{T} \backslash 0$ is such that $\pi \zeta=\bar{\mu}$ or $\pi \zeta=0$, then $u^{2} \in H^{2 s-n / 2+\varepsilon}(\lambda)$ for all $\varepsilon<\frac{1}{2}$. Theorem 1.7 of [14] yields $C^{\infty}$ regularity of $u^{2}$ in certain regions (see Figure $2 \mathrm{a}$ ), but that is not needed here.

We proceed to consider the term $R \beta\left(v R \beta u^{2}\right)$. In the above proof it was shown that $v R \beta u^{2} \in \mathscr{C}^{t}(\sigma)$ for $t<2 s-n / 2+\frac{1}{2}$. It is not hard to improve this to $t<2 s-n / 2+1$ by using, for example, the fact that $\widehat{\alpha v_{a 1}}=\widehat{\alpha}(\tau-|\xi, \eta|) \hat{f}_{1}(\xi, \eta)$ for $\alpha(t) \in C_{0}^{\infty}$. Theorem 2.6 would then give $R \beta\left(v R \beta u^{2}\right) \in \mathscr{H}^{t}(\sigma)$ for $t<$ $2 s-n / 2+2$, which is still not quite enough. The problem is that the local estimate $R \beta u^{2} \in H_{\text {loc }}^{2(s+1)-\alpha,-(s+1)+\alpha}\left(\Omega_{T}\right)$ is too imprecise. To find the extra regularity required, we shall extend certain pieces of $R \beta u^{2}$ across the boundary as solutions of wave equations in free space.

Lemma 3.36. In the case of Theorem 1.9(a), $R \beta\left(v R \beta u^{2}\right) \in H^{t}(\lambda)$ for all $t<$ $2 s-n / 2+\frac{5}{2}$, for all $\lambda \in \Lambda_{1}$.

Proof. For any $\bar{\mu} \in \stackrel{\circ}{C}$, let $\sigma=(0, \bar{\mu}) \in T^{*} b \Omega \backslash 0$ and observe that it will suffice to show

$$
v R \beta u^{2} \in H^{t}\left(\gamma_{-}(\sigma)\right) \cap \widetilde{H}^{0, t}(\sigma) \cap H^{t}\left(\gamma_{+}(\sigma)\right) \text { for } t<2 s-n / 2+\frac{3}{2},
$$

for then Theorem 2.6 implies $\widetilde{W F}_{2, t-1} R \beta\left(v R \beta u^{2}\right) \cap \gamma_{+}(\sigma)=\varnothing$ for such $t$.

Let $\widetilde{\Omega}_{T}=(-T, T) \times \mathbf{R}^{n}$ and denote by $C_{0}^{\infty}\left(\Omega_{T}\right)$ the space of restrictions to $\Omega_{T}$ of elements of $C_{0}^{\infty}\left(\widetilde{\Omega}_{T}\right)$. By finite propagation speed for mixed problems 
(e.g., [6, Theorem 8.5]) it is enough to prove (3.37) for $v R \beta \phi u^{2}$, for appropriate choices of $\phi(t, x, y) \in C_{0}^{\infty}\left(\Omega_{T}\right)$. So we fix such a $\phi$. With $\mu_{1}, \mu_{2}$ as in Lemma 3.28, choose $A_{1}(\tau, \eta), A_{2}(\tau, \eta) \in S^{0}\left(R^{n}\right)$ with $A_{i} \equiv 1$ on a small conic neighborhood of $\mu_{i}$ and such that $A_{i} \equiv 0$ outside a slightly larger cone contained in $\left\{\tau^{2}>|\eta|^{2}\right\}$. Writing $A\left(D^{\prime}\right)=A_{1}\left(D^{\prime}\right)+A_{2}\left(D^{\prime}\right)$ and $\phi u^{2}=A \phi u^{2}+$ $(1-A) \phi u^{2}$, we note that by Remark $3.35(1-A) \phi u^{2} \in H_{\text {loc }}^{t}\left(\Omega_{T}\right)$ for all $t<$ $2 s-n / 2+\frac{1}{2}$. Hence $R \beta(1-A) \phi u^{2} \in H_{\mathrm{loc}}^{t}\left(\Omega_{T}\right)$ for $t<2 s-n / 2+\frac{3}{2}$, which implies easily that $v R \beta(1-A) \phi u^{2}$ satisfies (3.37). Thus it is enough to consider $v R \beta A \phi u^{2}$. We concentrate now on $v R \beta A_{1} \phi u^{2}$, the other term being handled similarly. In fact, we may replace $A_{1}$ by $\widetilde{\phi} A_{1}$ where $\widetilde{\phi} \in C_{0}^{\infty}\left(\Omega_{T}\right)$ and $\widetilde{\phi} \equiv 1$ on supp $\phi$. Letting $\widetilde{\phi} A_{1} \phi=\widetilde{A}_{1}$, we proceed to find a good extension of $R \beta \widetilde{A}_{1} u^{2}$ across $x=0$.

The first step is to extend $\widetilde{A}_{1} u^{2}$. Choose $B\left(z, D^{\prime}\right)=b(z) \widetilde{B}\left(D^{\prime}\right)$, where $b \in C_{0}^{\infty}\left(\widetilde{\Omega}_{T}\right), \widetilde{B}(\tau, \eta) \in S^{0}$, and $\widetilde{B} \equiv 0$ outside a small conic neighborhood of $\mu_{1}$ contained in $\left\{\tau^{2}>|\eta|^{2}\right\}$, such that $B \equiv 1$ on ess supp $\widetilde{A}_{1}$. Now $\tilde{A}_{1} u^{2} \in H^{2 s-\alpha,-s+\alpha}(\Omega)$ for all $\alpha>\frac{1}{2}$. Fixing such an $\alpha$ close to $\frac{1}{2}$, we see that $(1-B) \widetilde{A}_{1} u^{2} \in H_{\text {loc }}^{2 s-\alpha, \infty}(\Omega)$, so it can be extended to an element $w_{1} \in H_{\text {loc }}^{2 s-\alpha, \infty}\left(\mathbf{R}^{n+1}\right)$. Next go back to $\widetilde{A_{1}} u^{2}$, extend it to an element $\left(\widetilde{A}_{1} u^{2}\right)_{e} \in$ $H^{2 s-\alpha,-s+\alpha}\left(\mathbf{R}^{n+1}\right)$, and set $w_{2}=B\left(\widetilde{A}_{1} u^{2}\right)_{e} \in H^{2 s-\alpha,-s+\alpha}\left(\mathbf{R}^{n+1}\right)$. Then $w=$ $w_{1}+w_{2}$ extends $\tilde{A}_{1} u^{2}$ and, after truncation in $x<0$ if necessary, has compact support. Note that if $\theta(\tau, \eta) \in S^{0}$ is such that cone $\operatorname{supp} \theta \cap$ cone $\operatorname{supp} \widetilde{B}$ $=\varnothing$, then $\theta\left(D^{\prime}\right) w \in H_{\text {loc }}^{2 s-\alpha, \infty}\left(\mathbf{R}^{n+1}\right) . R \beta \tilde{A}_{1} u^{2}$ can now be written

$$
E \beta w-C\left(\left.(E \beta w)\right|_{b \Omega}\right) \text {, }
$$

where the first term is a solution on $\mathbf{R}^{n+1}$ and the second remains to be extended.

We now show that $v E \beta w$ satisfies (3.37). Choose $\psi(z) \in C_{0}^{\infty}\left(\mathbf{R}^{n+1}\right)$ such that $\psi \equiv 1$ on a large neighborhood $\mathscr{O}$ of 0 , and choose $\chi(\tau, \eta) \in S^{0}$ such that $\chi \equiv 1$ on cone supp $\widetilde{B}$ and $\chi \equiv 0$ outside a slightly larger cone contained in $\left\{\tau^{2}>|\eta|^{2}\right\}$. We claim

$$
\left(1-\chi\left(D^{\prime}\right)\right) \psi E \beta w \in H_{\mathrm{loc}}^{2 s-\alpha+2}(\mathscr{O}) .
$$

To see this, write $\square(1-\chi) \psi E \beta w=(1-\chi) \psi \beta w+(1-\chi)[\square, \psi] E \beta w$. The first term is in $H_{\mathrm{loc}}^{2 s-\alpha, \infty}\left(\mathbf{R}^{n+1}\right)$ and the second vanishes on $\mathscr{O}$, so (3.39) follows from microlocal ellipticity, the fact that $\pi_{2} W F w \subset\{(\tau, \xi, \eta):(\tau, \eta) \in$ cone supp $\widetilde{B}$ or $(\tau, \eta)=0\}$, and our choice of $\chi$. (3.39) implies immediately that $v(1-\chi) \psi E \beta w$ satisfies (3.37) if $\mathscr{O}$ is large enough, so it remains to examine $v \chi\left(D^{\prime}\right) \psi E \beta w$. Since $\psi E \beta w \in H^{s+1}\left(\mathbf{R}^{n+1}\right)$ and $\square \psi E \beta w=$ $\psi \beta w+[\square, \psi] E \beta w \in H_{\text {comp }}^{s}\left(\mathbf{R}^{n+1}\right)$, it follows that

$$
\langle\tau, \xi, \eta\rangle^{s+1}\left\langle\tau-|\xi, \eta| \widehat{\psi E E \beta w} \in L^{2}\left(R^{n+1}\right) .\right.
$$


This permits us to use an argument similar to the proof of Lemma 3.28 on $v \chi \psi E \beta w$. Consider first $v_{a 2} \chi \psi E \beta w$. As before, for any $\alpha(t) \in C_{0}^{\infty}$,

$$
\widehat{\alpha v_{a 2}}=\gamma(\tau-|\xi, \eta|) \hat{h}(\tau, \xi, \eta) \quad \text { for some } \gamma \in \mathscr{S}, h \in H^{s}\left(\mathbf{R}^{n+1}\right) \text {. }
$$

Let $\chi_{2}$ be the characteristic function of a small conic neighborhood of $\left(1, \omega_{2}\right)$, and observe that it suffices to consider $\left(\chi_{2}(D) \alpha v_{a 2}\right)\left(\chi\left(D^{\prime}\right) \psi E \beta w\right)$. Denoting by $\theta(\mu)$ the characteristic function of a small conic neighborhood of $\bar{\mu}$ (disjoint from $\operatorname{supp} \chi$ ) contained in $\stackrel{\circ}{C}_{1}$, and, using (3.40), we shall apply (2.8) to

$$
G(\zeta, \beta)=\frac{\theta(\mu)\langle\mu\rangle^{2 s-n / 2+1+\varepsilon} \gamma\left(\tau_{0}-\left|\xi_{0}, \eta_{0}\right|\right) \chi\left(\mu-\mu_{0}\right) \chi_{2}(\beta)}{\langle\zeta-\beta\rangle^{s+1}\left\langle\tau-\tau_{0}-\left|\xi-\xi_{0}, \eta-\eta_{0}\right|\right\rangle\langle\beta\rangle^{s}} .
$$

On supp $G$ we have $|\zeta-\beta| \geq C|\beta|,\left|\mu_{0}\right| \geq C|\mu|$, and $\left|\mu-\mu_{0}\right| \geq C|\mu|$ so

$$
|G| \leq F=C \frac{\left|\gamma\left(\tau_{0}-\left|\xi_{0}, \eta_{0}\right|\right)\right| \chi\left(\mu-\mu_{0}\right) \chi_{2}(\beta)}{\langle\beta\rangle^{n / 2-\varepsilon}\left\langle\tau-\tau_{0}-\left|\xi-\xi_{0}, \eta-\eta_{0}\right|\right\rangle} .
$$

Choosing the map $T$ as in (3.31), we note that $\operatorname{det} d T>C>0$ since $-\eta_{01} /\left|\xi_{0}, \eta_{0}\right|>C>0$ and $\eta_{1}-\eta_{01} \geq 0$ on $\operatorname{supp} F$. Hence, as in the proof of Lemma 3.28, we conclude that $\int F^{2} d \beta<\infty$ if $\varepsilon<\frac{1}{2}$. Thus $v_{a 2} \chi \psi E \beta w$ satisfies (3.37). The remaining terms in $v \chi \psi E \beta w$ are either handled similarly or satisfy (3.37) trivially, so it follows finally that $v E \beta w$ satisfies (3.37).

To finish we must treat the second term in (3.38), $C\left(\left.(E \beta w)\right|_{b \Omega}\right)$. With $\chi$ and $\psi$ as before, it suffices by finite propagation speed to consider

$$
C\left(\left.(\psi E \beta w)\right|_{b \Omega}\right)=C\left(\left.(\chi \psi E \beta w)\right|_{b \Omega}\right)+C\left(\left.((1-\chi) \psi E \beta w)\right|_{b \Omega}\right) .
$$

Now (3.39) implies that the second term is in $H_{\text {loc }}^{2 s-\alpha+3 / 2}$ on a large neighborhood of 0 in $\Omega$, so $v C\left(\left.((1-\chi) \psi E \beta w)\right|_{b \Omega}\right)$ satisfies (3.37). Since cone supp $\chi(\mu)$ $C\left\{\tau^{2}>|\eta|^{2}\right\}$, the first term in the sum can be extended to $\mathbf{R}^{n+1}$ as a solution simply by letting $x$ take negative values in the integral formula (3.15). Call this extension $U$. By classical hyperbolic theory, since $\square \psi E \beta w \in H^{s}\left(\mathbf{R}^{n+1}\right)$ and $\psi E \beta w \in H^{s+1}\left(\mathbf{R}^{n+1}\right)$, it follows that $\left.(\chi \psi E \beta w)\right|_{b \Omega} \in H^{s+1}(b \Omega)$, and therefore $U \in H_{\mathrm{loc}}^{s+1}\left(\mathbf{R}^{n+1}\right)$. This together with $\square U=0$ implies $\square \psi U \in H_{\text {comp }}^{s}\left(R^{n+1}\right)$, and thus

$$
\langle\tau, \xi, \eta\rangle^{s+1}\langle\tau-|\xi, \eta|\rangle \widehat{\psi U} \in L^{2} .
$$

With (3.44) $v \psi U$ can be shown to satisfy (3.37) by the argument used above for $v \chi \psi E \beta w$, so this completes the proof of Lemma 3.36.

3.3. Proof of Theorem 1.9(b). Here $w$ as in 1.4(b) is used to define the Cauchy data in equation (1.2), so $\widehat{v_{a 1}}=e^{i t|\xi, \eta|} \hat{f}_{1}(\xi, \eta), \widehat{v_{a 2}}=e^{-i t|\xi, \eta|} \hat{f}_{2}(-\xi,-\eta)$, $\widehat{v_{b 1}}=e^{i t|\xi, \eta|} \hat{f}_{1}(-\xi, \eta)$, and $\widehat{v_{b 2}}=e^{-i t|\xi, \eta|} \hat{f}_{2}(\xi,-\eta)$. The appropriate analogues of Propositions 3.1 and 3.27 are obtained simply by replacing $\Lambda_{1}$ by $\Lambda_{2}$ in the statements. The above arguments, with a few changes that we shall now point 
out, also yield these new propositions. In what follows, by "Lemma $3.5^{\prime}$," for example, we mean a close analogue of Lemma 3.5 in the context of Theorem $1.9(\mathrm{~b})$, and similarly for $\left(3.7^{\prime}\right)$, Remark $3.26^{\prime}$, etc.

The main difference is that where we had ellipsoids there are now hyperboloids instead. For example, in Lemma $3.5^{\prime}$ let $S=\{\zeta:|\zeta| \geq M,|\zeta-\bar{\zeta}| \zeta|| \leq$ $\left.\varepsilon|\zeta|^{1 / \rho}\right\}$, where now we choose as a typical point to focus on, $\bar{\zeta}=2\left(1, \omega_{1}\right)-$ $\left(1, \omega_{2}\right)=(1,1 / \sqrt{2}, 3 / \sqrt{2}, 0) \in B^{\omega_{1},-\omega_{2}}$. Instead of (3.6) we must estimate

$\left(3.6^{\prime}\right) \int \hat{\beta}\left(\tau-\left|\xi-\xi_{0}, \eta-\eta_{0}\right|+\left|\xi_{0}, \eta_{0}\right|\right) \hat{f}_{1}\left(\xi-\xi_{0}, \eta-\eta_{0}\right) \hat{f}_{2}\left(-\xi_{0},-\eta_{0}\right) d \xi_{0} d \eta_{0}$.

The reader can easily verify that, corresponding to this choice of $\bar{\zeta}, 3.7^{\prime}$ and $3.8^{\prime}$ should be

$$
\begin{aligned}
& A=\bigcup_{\left|\varepsilon^{\prime}\right| \leq \delta^{\prime}}\left\{\left(\xi_{0}, \eta_{0}\right):|\xi, \eta| / \sqrt{5}+\delta|\xi, \eta|^{1 / \rho}+\varepsilon^{\prime}=\left|\xi-\xi_{0}, \eta-\eta_{0}\right|-\left|\xi_{0}, \eta_{0}\right|\right\}, \\
& \left.3.8^{\prime}\right) \quad B=\left\{\left(\xi_{0}, \eta_{0}\right):\left|\left(\xi_{0}, \eta_{0}\right)+\right| \xi, \eta\left|\omega_{2} / \sqrt{5}\right| \leq \varepsilon|\xi, \eta|^{1 / \rho}\right\} .
\end{aligned}
$$

Note that $A$ is a union of confocal half-hyperboloids with foci at $0,(\xi, \eta)$.

In the proof of Lemma 3.14' ${ }^{\prime}$, cone $\operatorname{supp} h(\tau, \eta)$ is concentrated near a point $\bar{\mu} \in \stackrel{\circ}{C}_{2}$. So again cone supp $h \subset\left\{\tau^{2}>|\eta|^{2}\right\}$, as required in formula (3.15).

In Remark $3.26^{\prime}$ it should be noted that the ray on $B^{\omega_{1},-\omega_{2}}$ and the ray on $B^{\omega_{1},-\omega_{2}^{*}}$ ( $\omega_{2}^{*}$ as before) which project to $\{t \bar{\mu}: t>0\}$ for a fixed $\bar{\mu} \in \stackrel{\circ}{C}_{2}$ now lie outside $\tau^{2}=|\xi, \eta|^{2}$. Again, the ray associated to the incoming-incoming interaction (the one on $B^{\omega_{1},-\omega_{2}}$ ) is closer to $\tau^{2}=|\xi, \eta|^{2}$.

In the proof of Lemma $3.28^{\prime},\left(3.30^{\prime}\right)$ should be

$$
C \frac{\left|\gamma\left(\tau-\tau_{0}-\left|\xi-\xi_{0}, \eta-\eta_{0}\right|\right) \widetilde{\gamma}\left(\tau_{0}+\left|\xi_{0}, \eta_{0}\right|\right)\right| \chi_{1}(\zeta-\beta) \chi_{2}(\beta)}{\langle\beta\rangle^{n / 2-\varepsilon}},
$$

where now $\chi_{1}, \chi_{2}$ are supported near $\left(1, \omega_{1}\right),-\left(1, \omega_{2}\right)$, respectively. Here one changes variables using $\left(T^{\prime}\right)^{-1}$, where $T^{\prime}:\left(\tau_{0}, \eta_{01}\right) \rightarrow\left(\tilde{\tau}, \tilde{\eta}_{1}\right)$ is the map

$$
\tilde{\tau}=\tau_{0}+\left|\xi_{0}, \eta_{0}\right|, \quad \tilde{\eta}_{1}=\tau-\tau_{0}-\left|\xi-\xi_{0}, \eta-\eta_{0}\right|,
$$

after noting that

$$
\operatorname{det}\left(d T^{\prime}\right)=\frac{\eta_{1}-\eta_{01}}{\left|\xi-\xi_{0}, \eta-\eta_{0}\right|}+\frac{\eta_{01}}{\left|\xi_{0}, \eta_{0}\right|} \geq C>0 \quad \text { on the support of }\left(3.30^{\prime}\right) .
$$

The arguments in the proof of Lemma 3.36 all go through with the obvious small changes to give Lemma 3.36' . For example, $\gamma\left(\tau_{0}-\left|\xi_{0}, \eta_{0}\right|\right)$ in (3.41) and (3.42) should be replaced by $\tilde{\gamma}\left(\tau_{0}+\left|\xi_{0}, \eta_{0}\right|\right)$ in $\left(3.41^{\prime}\right)$ and $\left(3.42^{\prime}\right)$.

\section{Self-SPREAding AT the Boundary}

In this section we prove Theorem $1.9(\mathrm{c})$. Here $w$ as in $(1.4)(\mathrm{c})$ is used to define the Cauchy data in (1.2), so in the notation of the introduction, 
$\widehat{v_{a 1}}=e^{i t|\xi, \eta|} \hat{g}(\xi, \eta), \widehat{v_{a 2}}=e^{-i t|\xi, \eta|} \hat{g}(-\xi,-\eta), \widehat{v_{b 1}}=e^{i t|\xi, \eta|} g(-\xi, \eta), \widehat{v_{b 2}}=$ $e^{-i t|\xi, \eta|} \hat{g}(\xi,-\eta)$. The first step in the analysis of $R \beta v^{2}$ is to get an appropriate lower bound for $\beta \widehat{v}_{a 1} v_{a 2}$ on a subconic neighborhood of each point in $B^{\tilde{\omega}}$. As in the proof of Theorem 1.9(b), this involves estimating the volume of a solid obtained by intersecting a family of confocal hyperboloids with a subconic region as in $(1.3)(\mathrm{c})$ (recall $\left(3.7^{\prime}\right)$ and $\left(3.8^{\prime}\right)$ ). In the present case this is slightly more difficult because, for example when $n=2$, each hyperbola in the family has an asymptote that is either parallel or nearly parallel to the axis of the subconic region (see Figure 3a), and this makes it easier for the hyperbolas to miss the region. But the main new difficulty arises in the analysis of the remainder $R \beta\left(u^{2}-v^{2}\right)$. Changes of variables such as those used in the two cases of crossing $\left((3.31)\right.$ and $\left.\left(3.31^{\prime}\right)\right)$ cannot be used to estimate antipodal products, because $\left|\operatorname{det} d T^{-1}\right|$ turns out to blow up. This problem is dealt with in the proofs of Lemmas 4.21 and 4.38 .

4.1. The singularities of $R \beta v^{2}$.

Proposition 4.1. Fix $\varepsilon>0$ arbitrarily small. Then if $\rho$ in (1.3) is taken close enough to $1, R \beta v^{2} \notin H^{2 s-n / 2+2+\varepsilon}(\lambda)$ for all $\lambda \in \Lambda_{3}$.

The proposition will follow easily once we show

$$
C\left(\left.\left(E \beta v_{a 1} v_{a 2}\right)\right|_{b \Omega}\right)-C\left(\left.\left(E \beta v_{a 1} v_{b 2}\right)\right|_{b \Omega}\right)
$$

has singularities as above on $\Lambda_{3}$. We shall fix $\bar{\mu}=(\sqrt{2 / 3}, \sqrt{1 / 3}, 0) \in \stackrel{\circ}{C_{3}}$ and find such singularities on $\gamma_{+}(0, \bar{\mu})$. The argument is the same for other $\mu \in \stackrel{\circ}{C_{3}}$. $B^{\tilde{\omega}}$ is the plane $\tau=\xi$, so $\bar{\zeta}=(\sqrt{2 / 3}, \sqrt{2 / 3}, \sqrt{1 / 3}, 0) \in B^{\tilde{\omega}}$ and $\pi \bar{\zeta}=\bar{\mu}$. If $H(\zeta)$ is the characteristic function of a small conic neighborhood of $\bar{\zeta}$, then since $\widehat{\beta v_{a 1} v_{a 2}}$ is rapidly decreasing outside $B^{\tilde{\omega}}[2$, Corollary 1.6], the proof of Lemma 3.3 shows that $W F\left[C\left(\left.\left(E \beta v_{a 1} v_{a 2}\right)\right|_{b \Omega}\right)-C\left(\left.U\right|_{b \Omega}\right)\right] \cap \gamma_{+}(0, \bar{\mu})=\varnothing$, where $\widehat{U}=\left(H(\zeta) /\left(\tau^{2}-|\xi, \eta|^{2}\right)\right) \widehat{\beta v_{a 1} v_{a 2}}$. So we proceed to study $\widehat{\left.U\right|_{b \Omega}}$.

For any $\omega \in S^{n-1}, \rho>1$, and $\alpha>0$, we will denote by $G_{\rho}(\omega, \alpha)$ the subconic set

$$
\left\{(\xi, \eta):|(\xi, \eta)-| \xi, \eta|\omega| \leq \alpha|\xi, \eta|^{1 / \rho}\right\}
$$

Lemma 4.3. There exist constants $\varepsilon, N$ such that on $S=\{\zeta:|\zeta| \geq N$, $\left.|\zeta-\bar{\zeta}| \zeta|| \leq \varepsilon|\zeta|^{2-\rho}\right\}$ we have $\widehat{\beta v_{a 1} v_{a 2}}(\zeta) \geq C\langle\zeta\rangle^{-(2 s+1+O(\rho-1))}$.

Proof. Choose an $\alpha<1$ as in (4.2). It suffices to prove the estimate for $\zeta \in S^{\prime}=\left\{\zeta:|(\xi, \eta)-(\sqrt{2 / 3}, \sqrt{1 / 3}, 0)| \xi, \eta|| \leq \varepsilon|\xi, \eta|^{2-\rho},|\tau-\sqrt{2 / 3}| \xi, \eta|| \leq\right.$ $\left.\varepsilon|\xi, \eta|^{2-\rho},|\xi, \eta| \geq N\right\}$. (Note that $2-\rho<1 / \rho$.) We must estimate

$$
\int \hat{\beta}\left(\tau-\left|\xi-\xi_{0}, \eta-\eta_{0}\right|+\left|\xi_{0}, \eta_{0}\right|\right) \hat{g}\left(\xi-\xi_{0}, \eta-\eta_{0}\right) \hat{g}\left(-\xi_{0},-\eta_{0}\right) d \xi_{0} d \eta_{0}
$$


So fix $\zeta=(\tau, \xi, \eta) \in S^{\prime}$, choose $\delta$ and $C$ such that $\widehat{\beta}(\sigma) \geq C$ for $|\sigma| \leq \delta$, and define

$$
A=\bigcup_{\left|\varepsilon^{\prime}\right| \leq \delta}\left\{\left(\xi_{0}, \eta_{0}\right): \tau+\varepsilon^{\prime}=\left|\xi-\xi_{0}, \eta-\eta_{0}\right|-\left|\xi_{0}, \eta_{0}\right|\right\},
$$

a union of confocal half-hyperboloids with foci at $0,(\xi, \eta)$. Noting that $G_{\rho}(-\widetilde{\omega}, \alpha)$ contains $\left\{\left(\xi_{0}, \eta_{0}\right):\left|\eta_{0}\right| \leq C_{1}\left|\xi_{0}\right|^{1 / \rho}, \xi_{0}<-C_{2}\right\}$ for some $C_{1}, C_{2}$ and letting $I_{M}$ denote the interval $\left[(-M-1)|\xi, \eta|^{\rho},-M|\xi, \eta|^{\rho}\right]$ for some large $M$ to be chosen, we define

$$
B=\left\{\left(\xi_{0}, \eta_{0}\right):\left|\eta_{0}\right| \leq C_{1}\left|\xi_{0}\right|^{1 / \rho}, \xi_{0} \in I_{M}\right\} .
$$

For fixed $M$ and $N$ sufficiently large, and $\varepsilon$ sufficiently small ( $\varepsilon, N$ as in the definition of $S^{\prime}$ ), the next lemma shows

$$
\operatorname{vol}(A \cap B) \geq C\langle\xi, \eta\rangle^{n-1+O(\rho-1)} \text { for a } C \text { independent of } \zeta \in S^{\prime} .
$$

The proof can be completed using (4.7) as follows. First we note that for $\left(\xi_{0}, \eta_{0}\right) \in B$ and $(\xi, \eta)$ as above, $\left(\xi-\xi_{0}, \eta-\eta_{0}\right) \in G_{\rho}(\widetilde{\omega}, 1)$. To see this, set $E=(\xi, \eta), E_{0}=\left(\xi_{0}, \eta_{0}\right)$ and write

$$
\begin{aligned}
\left|\frac{E-E_{0}}{\left|E-E_{0}\right|}-\widetilde{\omega}\right| & \leq\left|\frac{E_{0}-E}{\left|E-E_{0}\right|}-\frac{E_{0}}{\left|E-E_{0}\right|}\right|+\left|\frac{E_{0}}{\left|E-E_{0}\right|}-\frac{E_{0}}{\left|E_{0}\right|}\right|+\left|\frac{E_{0}}{\left|E_{0}\right|}-(-\widetilde{\omega})\right| \\
& =\mathrm{I}+\mathrm{II}+\mathrm{III} .
\end{aligned}
$$

Now III $\leq \alpha\left|\xi_{0}, \eta_{0}\right|^{1 / \rho-1}$, and since $\left|\xi-\xi_{0}, \eta-\eta_{0}\right| \sim\left|\xi_{0}, \eta_{0}\right|$, I and II are each $\leq C|\xi, \eta| /\left|\xi_{0}, \eta_{0}\right|$. Since $|\xi, \eta| \leq C\left(\left|\xi_{0}, \eta_{0}\right| / M\right)^{1 / \rho}$, the assertion follows if $M$ is large enough. So using (1.3)(c) and (4.7) we get for $\zeta \in S^{\prime}$,

$$
\begin{aligned}
\widehat{\beta v_{a 1} v_{a 2}} & \geq C \int_{A \cap B} \frac{d \xi_{0} d \eta_{0}}{\left\langle\xi-\xi_{0}, \eta-\eta_{0}\right\rangle^{s+n / 2+O(\rho-1)}\left\langle\xi_{0}, \eta_{0}\right\rangle^{s+n / 2+O(\rho-1)}} \\
& \geq C \frac{\langle\xi, \eta\rangle^{n-1+O(\rho-1)}}{\langle\xi, \eta\rangle^{(2 s+n+O(\rho-1)) \rho}},
\end{aligned}
$$

from which the lemma follows.

Lemma 4.10. With $A$ and $B$ as in (4.7), $\operatorname{vol}(A \cap B) \geq C\langle\xi, \eta\rangle^{n-1+O(\rho-1)}$ for a $C$ independent of $\zeta \in S^{\prime}$.

Proof. For convenience we suppose that $C_{1}=1$ in (4.6). We shall first consider the case $n=2$ and then explain the changes needed when $n>2$.

$n=2$. To each $\zeta \in S^{\prime},\left|\varepsilon^{\prime}\right| \leq \delta$, one can associate a half-hyperbola with foci at $0,(\xi, \eta)$ as in (4.5). The starting point of the proof is the observation that since $\bar{\zeta} \in B^{\tilde{\omega}}$, each such hyperbola has one asymptote parallel or nearly parallel to $\widetilde{\omega}=(1,0)$ (Figure 3a). Let the positive $x$-axis be as drawn in Figure 3a. If $r$ denotes distance from this axis, then the hyperbola associated to $\tau+\varepsilon^{\prime}, \xi, \eta$ consists of points satisfying $x^{2} / a^{2}-r^{2} / b^{2}=1$, where $2 a=\tau+\varepsilon^{\prime}$ and, with 
$2 c=|\xi, \eta|, b=\sqrt{c^{2}-a^{2}}$. Referring to Figure $3 a$ and using the definition of $S^{\prime}$, we obtain that $\left|\tan \phi-\sqrt{\frac{1}{2}}\right| \leq \varepsilon|\xi, \eta|^{1-\rho}$ and that $\tan \phi^{\prime}=b / a$ satisfies $\left|b / a-\sqrt{\frac{1}{2}}\right| \leq \varepsilon|\xi, \eta|^{1-\rho}$. Hence the nearly horizontal asymptote has slope $m$ satisfying $|m| \leq \varepsilon_{0}|\xi, \eta|^{1-\rho}$, with $\varepsilon_{0}$ independent of $\zeta \in S^{\prime},\left|\varepsilon^{\prime}\right| \leq \delta$. As $S^{\prime}$ shrinks, $\varepsilon_{0}$ can be taken smaller.

Next set $D=A \cap\left\{\left(\xi_{0}, \eta_{0}\right): \xi_{0} \in I_{M}\right\}$. We will show $D \subset B$ by considering asymptotes first. Let $\xi_{0}(t)=-(M+t)|\xi, \eta|^{\rho}, t \in[0,1]$. Referring to Figure $3 \mathrm{~b}$ we see that

$$
\begin{aligned}
u & =(M+t)^{1 / \rho}|\xi, \eta|, \text { while } v+w=\frac{\eta}{2} \pm \varepsilon_{0}|\xi, \eta|^{1-\rho}\left[(M+t)|\xi, \eta|^{\rho}+\frac{\xi}{2}\right] \\
& =\sqrt{\frac{1}{3}} \frac{|\xi, \eta|}{2} \pm \varepsilon|\xi, \eta|
\end{aligned}
$$

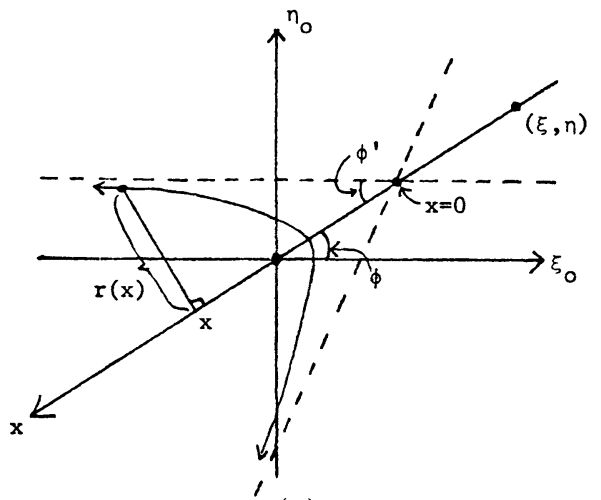

(a)

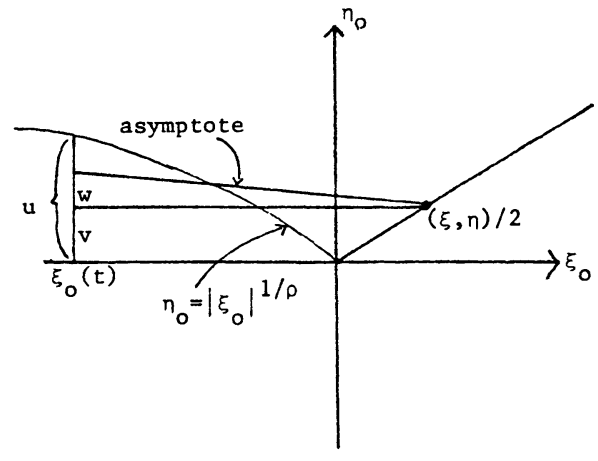

(b)

FIGURE 3

Thus each asymptote stays in $B$ over $I_{M}$. If $|\xi, \eta|$ is large, the same is true for the corresponding hyperbola, hence $D \subset B$. So to complete the proof it will suffice to show vol $D \geq C\langle\xi, \eta\rangle^{1+O(\rho-1)}$. For this we need:

Let $\Gamma$ be a small conic neighborhood of $\bar{\zeta}$. To each $(\tau, \xi, \eta) \in$ $\Gamma$ associate a hyperbola as above and let $r(x)$ be as in Figure 3a. Denote by $\Delta r(x)$ the magnitude of the change in $r(x)$ as $\tau$ increases to $\tau+\delta$. Then: (a) There exist constants $C_{1}, C_{2}$, $K$ such that for $x \geq|\xi, \eta| / 2>K$ we have $C_{1} \delta x / a \leq \Delta r(x) \leq$ $C_{2} \delta x / a$, where $a=\tau / 2$. Moreover, the same $C_{1}$ and $C_{2}$ work for all $\zeta \in \Gamma$ with $|\xi, \eta|>2 K$. (b) The same is true if $\delta$ is replaced by $|\xi, \eta|^{\varepsilon}$, where $\varepsilon<1$.

Assuming this for a moment we have, for some $M_{1}, M_{2}$,

$$
\operatorname{vol} D \geq \int_{M_{1}|\xi, \eta|^{\prime \prime}}^{M_{2}|\xi, \eta|^{\prime \prime}} C_{1} \frac{\delta x}{a} d x=\frac{C}{a} \delta|\xi, \eta|^{2 \rho} \geq C\langle\xi, \eta\rangle^{2 \rho-1}=C\langle\xi, \eta\rangle^{1+O(\rho-1)}
$$


since $a \sim|\xi, \eta|$.

To prove (4.12) we note that as $a$ increases to $\tilde{a}=a+\delta / 2, b$ decreases to $\tilde{b}$ satisfying $a^{2}+b^{2}=\tilde{a}^{2}+\tilde{b}^{2}\left(=c^{2}\right.$, where $\left.2 c=|\xi, \eta|\right)$. Thus there exist $K_{1}$, $K_{2}>0$ independent of $\zeta$ as in (4.12)(a) such that $b-K_{1} \delta<\tilde{b}<b-K_{2} \delta$. Since $\Delta r(x)=(b x / a) \sqrt{1-a^{2} / x^{2}}-(\tilde{b} x / \tilde{a}) \sqrt{1-\tilde{a}^{2} / x^{2}}$, one can replace $\tilde{b}$ by $b-K_{2} \delta$ to get the lower bound and by $b-K_{1} \delta$ to get the upper bound. $a=\sqrt{2 / 3}|\xi, \eta| / 2 \pm \varepsilon|\xi, \eta|$, so there is no problem with the square root. The same argument gives $(4.12)(\mathrm{b})$, which will be needed later. This completes the proof of Lemma 4.10 when $n=2$.

$n>2$. To each $\zeta \in S^{\prime},\left|\varepsilon^{\prime}\right| \leq \delta$ we now associate a half-hyperboloid as in (4.5). Let the $x$-axis be as shown in Figure $3 \mathrm{a}$, and in the $(n-1)$ dimensional space orthogonal to it, introduce spherical coordinates $(r, \theta)$, where $\theta=\left(\theta_{1}, \ldots, \theta_{n-2}\right)$. With $a, b$ as before, the hyperboloid satisfies $x^{2} / a^{2}-r^{2} / b^{2}=1$. Letting $P(\xi, \eta)$ denote the plane determined by the $\xi_{0}$-axis and $(\xi, \eta)$, we note that the intersection of $P(\xi, \eta)$ with the asymptote $r=b x / a, x>0$ (a cone) consists of two rays, one of which, by a previous argument, is parallel or nearly parallel to the $\xi_{0}$-axis. Choose $J_{M} \subset \subset I_{M}$ of length $\varepsilon_{1}|\xi, \eta|^{\rho}$, also centered at $\left(-M-\frac{1}{2}\right)|\xi, \eta|^{\rho}$, and set $D=A \cap\left\{\left(\xi_{0}, \eta_{0}\right): \xi_{0} \in J_{M}\right\} \cap P(\xi, \eta)$. Then $D \subset B$ by an argument in the case $n=2$, and all points in $D$ have the same $\theta$-coordinate $\bar{\theta}$. Next set $S_{x}=\left\{\theta:\left|\theta_{i}-\bar{\theta}_{i}\right|<\varepsilon_{2} x^{1-\rho}\right\}$ and for each $(\bar{\xi}, \bar{\eta}) \in D$ define $E(\bar{\xi}, \bar{\eta})=$ $\left\{\left(\xi_{0}, \eta_{0}\right): r\left(\xi_{0}, \eta_{0}\right)=r(\bar{\xi}, \bar{\eta}), x\left(\xi_{0}, \eta_{0}\right)=x(\bar{\xi}, \bar{\eta}), \theta\left(\xi_{0}, \eta_{0}\right) \in S_{x(\bar{\xi}, \bar{\eta})}\right\}$. Finally let $F=\bigcup_{(\bar{\xi}, \bar{\eta}) \in D} E(\bar{\xi}, \bar{\eta})$. Clearly $F \subset A$, and since $D \subset B$ it follows easily that if $\varepsilon_{1}$ and $\varepsilon_{2}$ are small enough, $F \subset B$. Letting $S_{x}^{\prime}$ be the subset of $S^{n-2}$ corresponding to $S_{x}$ and using (4.12)(a), we have for some $M_{1}, M_{2}$,

$$
\operatorname{vol}(A \cap B) \geq \operatorname{vol} F \geq \int_{M_{1}|\xi, \eta|^{\rho}}^{M_{2}|\xi, \eta|^{\prime \prime}} \int_{r(x)-C_{1} \delta x / a}^{r(x)} \int_{S_{x}^{\prime}} r^{n-2} d S d r d x .
$$

Since $\int_{S_{x}^{\prime}} d S=x^{O(\rho-1)}$ and $a \sim|\xi, \eta|$, integration gives

$$
\text { vol } F \geq C\langle\xi, \eta\rangle^{n-1+O(\rho-1)} \text {. }
$$

By the argument of Lemma 3.10, Lemma 4.3 implies

Lemma 4.15. There exist constants $\varepsilon, M$ such that for $\mu$ satisfying $|\mu| \geq M$, $|\mu-\bar{\mu}| \mu|| \leq \varepsilon|\mu|^{2-\rho}$ we have $\widehat{\left.U\right|_{b \Omega}} \leq-C\langle\mu\rangle^{-(2 s+2+O(\rho-1))}$.

The minus sign is there because $\tau^{2}-|\xi, \eta|^{2}<0$ on supp $H$. By the proof of Lemma 3.14, Lemma 4.15 implies

Lemma 4.16. Fix $\varepsilon>0$. If $\rho$ is chosen close enough to 1 , then for all $\lambda \in$ $\gamma_{+}(0, \bar{\mu}), C\left(\left.\left(E \beta v_{a 1} v_{a 2}\right)\right|_{b \Omega}\right) \notin H^{2 s-n / 2+2+\varepsilon}(\lambda)$.

Next consider $C\left(\left.\left(E \beta v_{a 1} v_{b 2}\right)\right|_{b \Omega}\right)$. We know that $\widehat{\beta v_{a 1} v_{b 2}}$ fails to be rapidly decreasing on the wedge determined by $(1,1,0)$ and $(-1,1,0)$, and so in 
particular on a subconic neighborhood $\Omega$ of $(0,1,0)$. Since $\pi \Omega$ is all of $(\tau, \eta)$-space, there is a possibility of complete cancellation of singularities in $C\left(\left.\left(E \beta v_{a 1} v_{a 2}\right)\right|_{b \Omega}\right)-C\left(\left.\left(E \beta v_{a 1} v_{b 2}\right)\right|_{b \Omega}\right)$ which must be ruled out to prove Proposition 4.1.

Lemma 4.17. Let $\widetilde{H}(\zeta)$ be the characteristic function of a small conic neighborhood of $(0,1,0)$ and set $\widehat{V}=\left(\widetilde{H}(\zeta) /\left(\tau^{2}-|\xi, \eta|^{2}\right)\right) \widehat{\beta v_{a 1} v_{b 2}}$. Then for any $\mu \in\left\{\tau^{2}>|\eta|^{2}\right\}$ such that $\mu \neq \pm t(1,0), t>0$ we have

$$
W F\left[C\left(\left.\left(E \beta v_{a 1} v_{b 2}\right)\right|_{b \Omega}\right)-C\left(\left.V\right|_{b \Omega}\right)\right] \cap \gamma_{+}(0, \mu)=\varnothing .
$$

Proof. The proof is essentially the same as that of Lemma 3.3.

Lemma 4.18. Fix $\varepsilon>0$. If $\rho$ is chosen close enough to 1 , then for all $\lambda \in \Lambda_{3}$, $C\left(\left.\left(E \beta v_{a 1} v_{a 2}\right)\right|_{b \Omega}\right)-C\left(\left.\left(E \beta v_{a 1} v_{b 2}\right)\right|_{b \Omega}\right) \notin H^{2 s-n / 2+2+\varepsilon}(\lambda)$.

Proof. Restricting attention to $\lambda \in \gamma_{+}(0, \bar{\mu})$ again, we note that in view of Lemma 4.17 and the proof of Lemma 4.16, it suffices to show that there exist constants $\varepsilon, M$ such that for $|\mu| \geq M,|\mu-\bar{\mu}| \mu|| \leq \varepsilon|\mu|^{2-\rho}$ we have

$$
\widehat{\left.U\right|_{b \Omega}}-\widehat{\left.V\right|_{b \Omega}} \leq-C\langle\mu\rangle^{-(2 s+2+O(\rho-1))} .
$$

Using Lemma 4.15 and the fact that $\widehat{\beta v_{a 1} v_{a 2}}$ is rapidly decreasing outside $B^{\grave{\omega}}$, one obtains this estimate by arguing just as in the proof of Lemma 3.18. The key point (see Remark 3.26) is that supp $H$ lies closer to $\tau^{2}=|\xi, \eta|^{2}$ than supp $\widetilde{H}$ does.

The comments in the paragraph following Remark 3.26 apply here also, so this concludes the proof of Proposition 4.1.

4.2. The remainder $R \beta\left(u^{2}-v^{2}\right)$. Theorem $1.9(\mathrm{c})$ follows immediately from Proposition 4.1 and the following proposition. Here we set $\widetilde{\mu}=\pi(1, \widetilde{\omega})=$ $(1,0)$.

Proposition 4.20. In the case of Theorem 1.9(c),

$$
R \beta\left(u^{2}-v^{2}\right) \in H^{2 s-n / 2+2+\varepsilon}(\lambda)
$$

for all $\varepsilon<\frac{1}{3}-\frac{2}{15}\left(n /\left(n+\frac{18}{5}\right)\right)$, for all $\lambda \in \Lambda_{3} \backslash\left\{\gamma_{+}(0, \tilde{\mu}) \cup \gamma_{+}(0,-\widetilde{\mu})\right\}$.

Recall that we must consider $R \beta\left(R \beta u^{2}\right)^{2}$ and $R \beta\left(v R \beta u^{2}\right)$. The results of $\S 2$ and the following lemma will allow us to handle the first term.

Lemma 4.21. If $\bar{\mu} \in \mathbf{R}^{n} \backslash 0$ lies outside a conic neighborhood of $\{\tilde{\mu},-\tilde{\mu}\}$, then for $\sigma=(t, y, \bar{\mu}) \in T^{*} b \Omega \backslash 0, v^{2} \in \widetilde{H}^{2 s-n / 2+\varepsilon}(\sigma)$ for all $\varepsilon<\frac{1}{2}-n / 4(n+2)$. If

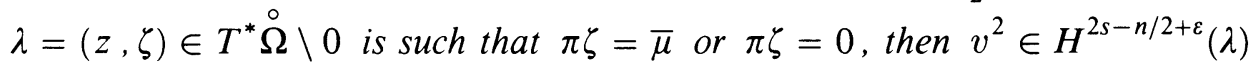
for such $\varepsilon$.

Proof. It will suffice to consider $v_{a 1} v_{a 2}$ and $v_{a 2} v_{b 1}$, since the remaining terms either can be treated similarly or satisfy the conditions of the lemma trivially. 
$v_{a 2} v_{b 1}$. Since $\widehat{\beta v_{a 2} v_{b 1}}$ is rapidly decreasing outside the wedge determined by $(1,-1,0)$ and $(-1,-1,0)$, it is tempting to conclude that $v_{a 2} v_{b 1}$ satisfies the above conditions trivially. However, since rapid decrease fails on a subconic neighborhood $\Omega$ of $(0,-1,0)$ and $\pi \Omega$ is all of $(\tau, \eta)$-space, there is something to prove.

For any $\alpha(t) \in C_{0}^{\infty}, \widehat{\alpha v_{b 1}}=\gamma(\tau-|\xi, \eta|) \hat{h}_{1}(\zeta)$ and $\widehat{\alpha v_{a 2}}=\tilde{\gamma}(\tau+|\xi, \eta|) \hat{h}_{2}(\zeta)$ for some $\gamma, \tilde{\gamma} \in \mathscr{P}$ and $h_{1}, h_{2} \in H^{s}\left(\mathbf{R}^{n+1}\right)$. Let $\chi_{1}, \chi_{2}$ be characteristic functions of small conic neighborhoods of $(1,-1,0),-(1,1,0)$, respectively, and let $\chi(\mu)$ be the characteristic function of a small conic neighborhood of $\bar{\mu}$. It is clearly enough to examine $\left(\chi_{1}(D) \alpha v_{b 1}\right)\left(\chi_{2}(D) \alpha v_{a 2}\right)$, and for this we apply $(2.8)$ to

$$
G(\zeta, \beta)=\frac{\chi(\mu)\langle\zeta\rangle^{2 s-n / 2+\varepsilon} \gamma\left(\tau-\tau_{0}-\left|\xi-\xi_{0}, \eta-\eta_{0}\right|\right) \tilde{\gamma}\left(\tau_{0}+\left|\xi_{0}, \eta_{0}\right|\right) \chi_{1}(\zeta-\beta) \chi_{2}(\beta)}{\langle\zeta-\beta\rangle^{s}\langle\beta\rangle^{s}} .
$$

The required estimates can be shown to hold for all $\varepsilon<\frac{1}{2}$ by an argument almost identical to the proof of Lemma 3.28. The only difference is that here one changes variables using the map $T^{-1}$, where $T:\left(\tau_{0}, \xi_{0}\right) \rightarrow(\widetilde{\tau}, \widetilde{\xi})$ is given by

$$
\tilde{\tau}=\tau_{0}+\left|\xi_{0}, \eta_{0}\right|, \quad \tilde{\xi}=\tau-\tau_{0}-\left|\xi-\xi_{0}, \eta-\eta_{0}\right|,
$$

after noting that

$$
\operatorname{det}(d T)=\frac{\xi-\xi_{0}}{\left|\xi-\xi_{0}, \eta-\eta_{0}\right|}+\frac{\xi_{0}}{\left|\xi_{0}, \eta_{0}\right|}<C<0 \quad \text { on supp } G .
$$

To prove the assertion of the lemma for $\lambda=(z, \bar{\zeta})$ with $\pi \bar{\zeta}=0$ simply replace $\chi(\mu)$ by the characteristic function of a small conic neighborhood of $\bar{\zeta}$.

$v_{a 1} v_{a 2}$. With $\chi_{2}$ as above but now taking $\chi_{1}$ to be the characteristic function of a small conic neighborhood of $(1,1,0)$, we need to consider

$$
\left(\chi_{1}(D) \alpha v_{a 1}\right)\left(\chi_{2}(D) \alpha v_{a 2}\right) \text {. }
$$

The corresponding function $G(\zeta, \beta)$ to which we shall apply $(2.8)$ then has the same appearance as (4.22). It will be convenient to suppose that $\chi_{2}$ has been multiplied by the characteristic function $\chi_{2}^{\prime}\left(\xi_{0}, \eta_{0}\right)$ of a small conic neighborhood of $-\tilde{\omega}$. Also we take $\chi(\mu)$ to be the characteristic function of a small conic neighborhood of $\bar{\mu}=(\sqrt{2 / 3}, \sqrt{1 / 3}, 0)$, the argument being the same for other choices of $\bar{\mu}$. Note that we cannot use a change of variables such as (4.23) in this case, since $\left|\operatorname{det} d T^{-1}\right|$ blows up on $\operatorname{supp} G$.

Now $\bar{\mu}=\pi \bar{\zeta}$ where $\bar{\zeta}=(\sqrt{2 / 3}, \sqrt{2 / 3}, \sqrt{1 / 3}, 0) \in B^{\tilde{\omega}}$. Let $A$ be a small conic neighborhood of $\bar{\zeta}, B=A^{c}$, and $\chi_{A}, \chi_{B}$ the corresponding characteristic functions. $\chi_{B}(\zeta) G$ satisfies the required estimates for any $\varepsilon$ since $\widehat{\beta v_{a 1} v_{a 2}}$ is rapidly decreasing away from $B^{\check{\omega}}$, so it remains to consider $\chi_{A} G$. For $(\tau, \xi, \eta) \in A$ and any small $\varepsilon^{\prime}>0$ define

$$
S=\left\{\left(\tau_{0}, \xi_{0}, \eta_{0}\right):\left|\tau-\tau_{0}-\right| \xi-\xi_{0}, \eta-\eta_{0}|| \leq|\xi, \eta|^{\varepsilon^{\prime}},\left|\tau_{0}+\right| \xi_{0}, \eta_{0}|| \leq|\xi, \eta|^{\varepsilon^{\prime}}\right\} .
$$


Since the required estimates obviously hold if either inequality in (4.25) is reversed, we can reduce to considering

$$
\begin{aligned}
& \int_{S \cap \text { supp } \chi_{2}}\left|\chi_{A} G\right|^{2} d \beta \\
& \quad \leq C \chi_{A} \int_{S \cap \operatorname{supp} \chi_{2}}\left(\frac{\langle\xi, \eta\rangle^{2 s-n / 2+\varepsilon}}{\left\langle\xi_{0}, \eta_{0}\right\rangle^{2 s}}\right)^{2}\left|\tilde{\gamma}^{2}\left(\tau_{0}+\left|\xi_{0}, \eta_{0}\right|\right)\right| d \tau_{0} d \xi_{0} d \eta_{0},
\end{aligned}
$$

where we have used $|\zeta-\beta| \geq C|\beta| \geq C\left|\xi_{0}, \eta_{0}\right|$ on $\operatorname{supp} G$. Let

$$
S^{\prime}=\left\{\left(\xi_{0}, \eta_{0}\right):|\tau-| \xi-\xi_{0}, \eta-\eta_{0}|+| \xi_{0}, \eta_{0}|| \leq 2|\xi, \eta|^{\varepsilon^{\prime}}\right\} .
$$

Setting $\tilde{\tau}=\tau_{0}+\left|\xi_{0}, \eta_{0}\right|$ and integrating in $\tilde{\tau}$, we obtain that the right side of $(4.26)$ is $\leq$

$$
\begin{aligned}
\chi_{A} C & \int_{S^{\prime} \cap \operatorname{supp} \chi_{2}^{\prime}\left(\xi_{0}, \eta_{0}\right)}\left(\frac{\langle\xi, \eta\rangle^{2 s-n / 2+\varepsilon}}{\left\langle\xi_{0}, \eta_{0}\right\rangle^{2 s}}\right)^{2} d \xi_{0} d \eta_{0} \\
& =\chi_{A} \int_{D_{1}}|F|^{2} d \xi_{0} d \eta_{0}+\chi_{A} \int_{D_{2}}|F|^{2} d \xi_{0} d \eta_{0}
\end{aligned}
$$

where

$$
\begin{aligned}
& D_{1}=S^{\prime} \cap \operatorname{supp} \chi_{2}^{\prime}\left(\xi_{0}, \eta_{0}\right) \cap\left\{\left|\xi_{0}, \eta_{0}\right| \geq|\xi, \eta|^{M}\right\}, \\
& D_{2}=S^{\prime} \cap \operatorname{supp} \chi_{2}^{\prime}\left(\xi_{0}, \eta_{0}\right) \cap\left\{\left|\xi_{0}, \eta_{0}\right| \leq|\xi, \eta|^{M}\right\},
\end{aligned}
$$

for some $M>1$ to be chosen. For $F$ restricted to $D_{1}$ we have

$$
F=\frac{C\langle\xi, \eta\rangle^{2 s-n / 2+\varepsilon}}{\left\langle\xi_{0}, \eta_{0}\right\rangle^{n / 2+\delta}\left\langle\xi_{0}, \eta_{0}\right\rangle^{2 s-n / 2-\delta}} \leq \frac{C\langle\xi, \eta\rangle^{2 s-n / 2+\varepsilon}}{\left\langle\xi_{0}, \eta_{0}\right\rangle^{n / 2+\delta}\langle\xi, \eta\rangle^{(2 s-n / 2-\delta) M}}
$$

for any small $\delta>0$. Thus $\left.F\right|_{D_{1}}$ satisfies the conditions of (2.8) provided

(4.30) $2 s-n / 2+\varepsilon \leq(2 s-n / 2-\delta) M$, that is, $\varepsilon \leq-\delta+(2 s-n / 2-\delta)(M-1)$.

Next we consider the integral on $D_{2}$. Note first that $S^{\prime}=\bigcup_{|\alpha| \leq 2}\left\{\left(\xi_{0}, \eta_{0}\right): \tau+\right.$ $\left.\alpha|\xi, \eta|^{\varepsilon^{\prime}}=\left|\xi-\xi_{0}, \eta-\eta_{0}\right|-\left|\xi_{0}, \eta_{0}\right|\right\}$, a union of confocal half-hyperboloids with foci at $0,(\xi, \eta)$. Since $(\tau, \xi, \eta) \in A$, a small conic neighborhood of $\bar{\zeta} \in B^{\check{\omega}}$, all points in $S^{\prime} \cap \operatorname{supp} \chi_{2}^{\prime}\left(\xi_{0}, \eta_{0}\right)$ must satisfy $\left|\xi_{0}, \eta_{0}\right| \geq N|\xi, \eta|$ for an $N$ that can be taken larger as $A$ shrinks. (By an argument in the proof of Lemma 4.10 we know, for example when $n=2$, that each hyperbola has one nearly horizontal asymptote.) This implies
(a) $\chi_{A} \int_{D_{2}}|F|^{2} d \xi_{0} d \eta_{0} \leq C \operatorname{vol}\left(D_{2}\right) /\langle\xi, \eta\rangle^{n-2 \varepsilon}$ and
(b) $\operatorname{vol}\left(D_{2}\right) \leq \operatorname{vol}\left(S^{\prime} \cap\left\{\left(\xi_{0}, \eta_{0}\right): N|\xi, \eta| \leq\left|\xi_{0}, \eta_{0}\right| \leq|\xi, \eta|^{M}, \xi_{0}<0\right\}\right)$.

With $2 a=\tau$ and $b=\sqrt{c^{2}-a^{2}}$, where $2 c=|\xi, \eta|$, the half-hyperboloid $\tau=\left|\xi-\xi_{0}, \eta-\eta_{0}\right|-\left|\xi_{0}, \eta_{0}\right|$ satisfies $x^{2} / a^{2}-r^{2} / b^{2}=1$ in the coordinates introduced in the proof of Lemma 4.10 (see Figure 3a). Thus, using (4.31)(b) 
and (4.12)(b) (which applies equally well to hyperboloids), we have for some $C_{n}, N_{1}, N_{2}, C$ independent of $(\tau, \xi, \eta) \in A$ and with $\Delta r(x)=C|\xi, \eta|^{\varepsilon^{\prime}} x / a$ :

$$
\operatorname{vol}\left(D_{2}\right) \leq C_{n} \int_{N_{1}|\xi, \eta|}^{N_{2}|\xi, \eta|^{M}} \int_{r(x)-\Delta r(x)}^{r(x)+\Delta r(x)} r^{n-2} d r d x
$$

where $r(x)=(b x / a) \sqrt{1-a^{2} / x^{2}} \leq C^{\prime} x$ for a $C^{\prime}$ independent of $(\tau, \xi, \eta) \in A$. Since $a \sim|\xi, \eta|$ the right side of (4.32) is

$$
\leq C_{n} \int_{N_{1}|\xi, \eta|}^{N_{2}|\xi, \eta|^{1 \prime}} x \frac{|\xi, \eta|^{\varepsilon^{\prime}} x}{a} d x \leq C_{n}^{\prime}|\xi, \eta|^{n M-1+\varepsilon^{\prime}}
$$

With (4.31) this gives

$$
\chi_{A} \int_{D_{2}}|F|^{2} d \xi_{0} d \eta_{0} \leq C \frac{\langle\xi, \eta\rangle^{n M-1+\varepsilon^{\prime}}}{\langle\xi, \eta\rangle^{n-2 \varepsilon}}=C\langle\xi, \eta\rangle^{-\left(n+1-n M-\varepsilon^{\prime}-2 \varepsilon\right)}
$$

So to apply $(2.8)$ we need

$$
\varepsilon \leq\left(n+1-n M-\varepsilon^{\prime}\right) / 2 .
$$

To get the largest $\varepsilon$ satisfying both (4.30) and (4.35) for all $s>(n+2) / 2$, one should take $M=\left(n+\frac{5}{2}\right) /(n+2)$. Then any fixed $\varepsilon<\frac{1}{2}-n / 4(n+2)$ satisfies both (4.30) and (4.35) provided $\delta$ and $\varepsilon^{\prime}$ are taken small enough, as we are free to do.

Lemma 4.36. $v^{2}$ can be replaced by $u^{2}$ in the statement of Lemma 4.21 .

Proof. This follows from an argument almost exactly like that used to establish (3.34) in the proof of Lemma 3.33. The only difference is that here one must make full use of Theorems 2.6 and 2.7, instead of using just the results on propagation near hyperbolic points.

Lemma 4.37. In the case of Theorem 1.9(c), $R \beta\left(R \beta u^{2}\right)^{2} \in H^{2 s-n / 2+2+\varepsilon}(\lambda)$ for all $\varepsilon<\frac{1}{2}-n / 4(n+2)$, for all $\lambda \in \Lambda_{3} \backslash\left\{\gamma_{+}(0, \tilde{\mu}) \cup \gamma_{+}(0,-\tilde{\mu})\right\}$, where $\widetilde{\mu}=$ $\pi(1, \widetilde{\omega})=(1,0)$.

Proof. This follows from the proof of Lemma 3.33, with (3.34) replaced by (a special case of) Lemma 4.36.

Lemma 4.38. In the case of Theorem $1.9(\mathrm{c}), R \beta\left(v R \beta u^{2}\right) \in H^{2 s-n / 2+2+\varepsilon}(\lambda)$ for all $\varepsilon<\frac{1}{3}-\frac{2}{15}\left(n /\left(n+\frac{18}{5}\right)\right)$, for all $\lambda \in \Lambda_{3} \backslash\left\{\gamma_{+}(0, \tilde{\mu}) \cup \gamma_{+}(0,-\tilde{\mu})\right\}$, where $\widetilde{\mu}=(1,0)$.

Proof. With $\sigma=(0, \bar{\mu})$, where $\bar{\mu}=\pi \bar{\zeta}, \bar{\zeta}=(\sqrt{2 / 3}, \sqrt{2 / 3}, \sqrt{1 / 3}, 0) \in B^{\check{\omega}}$, it suffices in view of Theorem 2.6 to show

$$
v R \beta u^{2} \in H^{2 s-n / 2+1+\varepsilon}\left(\gamma_{-}(\sigma) \cup \gamma_{+}(\sigma)\right) \cap \tilde{H}^{0,2 s-n / 2+1+\varepsilon}(\sigma) \text { for } \varepsilon \text { as above , }
$$

the proof being the same for other $\sigma$. The preliminary reductions and extension argument are almost identical to those in the proof of Lemma 3.36. Now, of 
course, $A_{1}(\mu), A_{2}(\mu) \in S^{0}$ are chosen $\equiv 1$ and with conic support near $\tilde{\mu}$, $-\widetilde{\mu}$, respectively. Again we reduce to considering $v R \beta \widetilde{A}_{1} u^{2}$, where $\widetilde{A}_{1}=\widetilde{\phi} A_{1} \phi$ for appropriate $\phi, \widetilde{\phi} \in C_{0}^{\infty}\left(\Omega_{T}\right)$. Note that Lemma 4.36 now plays the role of Remark 3.35 in that reduction argument. Again $R \beta \widetilde{A}_{1} u^{2}$ can be written

$$
E \beta w-C\left(\left.(E \beta w)\right|_{b \Omega}\right),
$$

where $w$ is an extension of $\tilde{A}_{1} u^{2}$ defined as before. With $\chi(\mu) \in S^{0}$ chosen as in the proof of Lemma 3.36 ( $\equiv 1$ and with conic support near $\tilde{\mu}$ ) and $\psi(z) \in C_{0}^{\infty}\left(\mathbf{R}^{n+1}\right)$ such that $\psi \equiv 1$ on a large neighborhood $\mathscr{O} \ni 0$, we reduce as before to considering $v \chi\left(D^{\prime}\right) \psi E \beta w$ and $v C\left(\left.(\chi \psi E \beta w)\right|_{b \Omega}\right)$, where

$$
\langle\tau, \xi, \eta\rangle^{s+1}\left\langle\tau-|\xi, \eta| \widehat{\psi E \beta w} \in L^{2}\left(\mathbf{R}^{n+1}\right) .\right.
$$

First we examine $v_{a 2} \chi\left(D^{\prime}\right) \psi E \beta w$. Choose $\chi_{i}(\zeta) \in S^{0}, i=1,2,3$, such that $\sum \chi_{i}=\chi(\mu)$, with $\chi_{1}, \chi_{2} \equiv 1$ and having conic support near $(1,1,0)$, $(1,-1,0)$ respectively. Let $\chi_{a 2}(\zeta)$ be the characteristic function of a small conic neighborhood of $-(1,1,0)$ and $\theta(\mu)$ the characteristic function of a small conic neighborhood of $\bar{\mu}$. We proceed to consider the terms $v_{a 2} \chi_{i}(D) \psi E \beta w$ in turn.

$v_{a 2} \chi_{1} \psi E \beta w$ can be handled much as $v_{a 1} v_{a 2}$ was in the proof of Lemma 4.21. It is enough to estimate $\left(\chi_{a 2}(D) \alpha(t) v_{a 2}\right)\left(\chi_{1}(D) \psi E \beta w\right)$ for $\alpha \in C_{0}^{\infty}$, and this leads us, using (4.41), to apply (2.8) to

$$
G(\zeta, \beta)=\frac{\theta(\mu)\langle\zeta\rangle^{2 s-n / 2+1+\varepsilon} \gamma\left(\tau_{0}+\left|\xi_{0}, \eta_{0}\right|\right) \chi_{1}(\zeta-\beta) \chi_{a 2}(\beta)}{\langle\zeta-\beta\rangle^{s+1}\left\langle\tau-\tau_{0}-\left|\zeta-\xi_{0}, \eta-\eta_{0}\right|\right\rangle\langle\beta\rangle^{s}},
$$

where $\gamma \in \mathscr{S}$. Let $A$ be a small conic neighborhood of $\bar{\zeta}, B=A^{c}$, and $\chi_{A}$, $\chi_{B}$ the corresponding characteristic functions. Observe that if $\left|\tau-\tau_{0}-\right| \xi-$ $\xi_{0}, \eta-\eta_{0}|| \geq|\xi, \eta|^{\varepsilon^{\prime}}$ for some $\varepsilon^{\prime}>0$, then since $|\zeta-\beta| \geq C|\beta| \geq C^{\prime}|\zeta|$ and $\langle\zeta\rangle \sim\langle\xi, \eta\rangle$ on $\operatorname{supp} \chi_{A}(\zeta) G$,

$$
\left|\chi_{A} G\right| \leq \frac{C\left|\gamma\left(\tau_{0}+\left|\xi_{0}, \eta_{0}\right|\right)\right|}{\left\langle\xi_{0}, \eta_{0}\right\rangle^{n / 2-\varepsilon+\varepsilon^{\prime}}} \in L^{2}\left(R^{n+1}\right) \quad \text { as long as } \varepsilon<\varepsilon^{\prime} .
$$

This allows us to treat $\chi_{A} G$ by showing

$$
\sup _{\zeta} \int_{S \cap \operatorname{supp} \chi_{u 2}}\left|\chi_{A} G\right|^{2} d \beta<\infty,
$$

where $S$ is defined as in (4.25). Arguing exactly as in the proof of Lemma 4.21, we find that $\varepsilon$ must satisfy the following conditions in order for (4.44) to hold:

(4.45) (a) $\varepsilon \leq-\delta+(2 s+1-n / 2-\delta)(M-1)$ (corresponding to 4.30$)$,

(b) $\varepsilon \leq\left(n+1-n M-\varepsilon^{\prime}\right) / 2$ (as in 4.35$)$,

(c) $\varepsilon<\varepsilon^{\prime}$ (from 4.43 ).

Here $\delta$ can be as small as desired and $M>1$ remains to be chosen. To get the largest $\varepsilon$ satisfying (4.45) for all $s>(n+2) / 2$, one should take 
$M=\left(\frac{5}{6} n+\frac{10}{3}\right) /\left(\frac{5}{6} n+3\right)$. Then for any $\varepsilon<\frac{1}{3}-\frac{2}{15}\left(n /\left(n+\frac{18}{5}\right)\right)$ there exist $\varepsilon^{\prime}>\varepsilon$ such that (4.45) holds, provided $\delta$ is taken small enough.

Next consider $\chi_{B} G$, which has $\zeta$-support away from $B^{\tilde{\omega}}$ if cone $\operatorname{supp} \theta(\mu)$ is small enough, as we are free to arrange. The following result of M. Beals [2, Lemma 1.5(iii)] is useful here. (It is, we should note, the key estimate in the proof of the $3 s$-theorem.)

Let $K_{ \pm}$be small conic neighborhoods of $\pm(1, \widetilde{\omega})$, respectively. Suppose $n / 2<s_{1} \leq s_{2}, r_{i}>s_{i}+\frac{1}{2}$ and that $u_{ \pm}$have the properties $\pi_{2} W F u_{ \pm} \subset K_{ \pm},\langle\zeta\rangle^{s_{1}}\langle\tau-|\xi, \eta|\rangle^{r_{1}-s_{1}} \hat{u}_{+} \in L^{2}$, $\langle\zeta\rangle^{s_{2}}\langle\tau+|\xi, \eta|\rangle^{r_{2}-s_{2}} \hat{u}_{-} \in L^{2}$. Then if $E$ is a small conic neighborhood of a point $\lambda \notin B^{\tilde{\omega}}$ and $\chi_{E}$ the characteristic function of $E$, we have $\langle\zeta\rangle^{t} \chi_{E}(\zeta){\widehat{u_{+}}}_{-} \in L^{2}$ for all $t<$ $\min \left(s_{1}+r_{2}-n / 2, s_{2}+r_{1}-n / 2\right)$, provided $K_{ \pm}$are sufficiently small.

We now apply (4.46) with $u_{+}=\chi_{1}(D) \psi E \beta w, u_{-}=\chi_{a 2}(D) \alpha(t) v_{a 2}$. Note that we can take $s_{2}=s, r_{2}$ arbitrarily large, and by (4.41), $s_{1}=s+1, r_{1}=$ $s+2$. It follows that $\langle\zeta\rangle^{2 s-n / 2+1+\varepsilon} \theta(\mu) \chi_{B}(\zeta){\widehat{u_{+}}}_{u_{-}} \in L^{2}$ for all $\varepsilon<1$ provided cone supp $\chi_{1}$, cone supp $\chi_{a 2}$ are sufficiently small. (Alternatively, one can apply the proof of 4.46 to show that $\chi_{B} G$ satisfies the conditions of (2.8).)

We turn now to $v_{a 2} \chi_{2}(D) \psi E \beta w$. The corresponding $G(\zeta, \beta)$ looks just like (4.42) with $\chi_{2}$ in place of $\chi_{1}$. With $\left\langle\tau-\tau_{0}-\left|\xi-\xi_{0}, \eta-\eta_{0}\right|\right\rangle^{-1}$ playing the role of $\gamma\left(\tau-\tau_{0}-\left|\xi-\xi_{0}, \eta-\eta_{0}\right|\right)$ (as in (4.22)), the argument used for $v_{a 2} v_{b 1}$ in the proof of Lemma 4.21 shows that $G$ satisfies the conditions of (2.8) for any $\varepsilon<\frac{1}{2}$.

It remains to consider $v_{a 2} \chi_{3}(D) \psi E \beta w$. Recall that $\psi E \beta w \in H^{s+1}\left(R^{n+1}\right)$ and that $\square \psi E \beta w \in H^{s}\left(\mathbf{R}^{n+1}\right)$. Since $\pi_{2} W F(E \beta w) \cap$ char $\square$ is concentrated near $\{(1,1,0),(1,-1,0)\}$, it follows from microlocal ellipticity and the fact that $\chi_{3} \equiv 0$ near these points that $\chi_{3} \psi E \beta w \in H^{s+2}\left(\mathbf{R}^{n+1}\right)$. Thus we can take

$$
G(\zeta, \beta)=\frac{\theta(\mu)\langle\mu\rangle^{2 s-n / 2+1+\varepsilon} \gamma\left(\tau_{0}+\left|\xi_{0}, \eta_{0}\right|\right) \chi_{3}(\zeta-\beta) \chi_{a 2}(\beta)}{\langle\zeta-\beta\rangle^{s+2}\langle\beta\rangle^{s}}
$$

Since $|\zeta-\beta| \geq C|\zeta|$ and $\left|\mu_{0}\right| \geq C|\mu|$ on $\operatorname{supp} G \quad(|\beta| \geq C|\zeta|$ does not hold here),

$$
|G| \leq C \frac{\left|\gamma\left(\tau_{0}+\left|\xi_{0}, \eta_{0}\right|\right)\right|}{\langle\beta\rangle^{n / 2+1-\varepsilon}} \leq C \frac{\left|\gamma\left(\tau_{0}+\left|\xi_{0}, \eta_{0}\right|\right)\right|}{\left\langle\xi_{0}, \eta_{0}\right\rangle^{n / 2+1-\varepsilon}} \in L^{2}\left(\mathbf{R}^{n+1}\right) \quad \text { for } \varepsilon<1
$$

This finishes the proof that $v_{a 2} \chi\left(D^{\prime}\right) \psi E \beta w$ satisfies (4.39). Since the other terms in $v \chi \psi E \beta w$ are either similar or satisfy (4.39) trivially, it remains only to examine $v C\left(\left.(\chi \psi E \beta w)\right|_{b \Omega}\right)$. Just as in the proof of Lemma 3.36, after extending $C\left(\left.(\chi \psi E \beta w)\right|_{b \Omega}\right)$ across $\chi=0$ as a solution, the product with $v$ can be shown to satisfy (4.39) by the argument used for $v \chi \psi E \beta w$. 
Lemmas 4.37 and 4.38 together imply Proposition 4.20, so the proof of Theorem $1.9(\mathrm{c})$ is complete.

\section{OPEN PROBLEMS}

5.1. Self-spreading of a gliding ray. It is interesting to consider what happens when the transversality hypothesis is removed in Problem A and Problem B. Let $\bar{\omega}=(\bar{\xi}, \bar{\eta})=(0,1,0)$ and suppose $u \in H_{\mathrm{loc}}^{s}\left(\Omega_{T}\right)$ satisfies

$$
\begin{gathered}
\square u=\beta f(u),\left.\quad u\right|_{b \Omega_{T}} \in C^{\infty}, \\
\left.W F_{b} u\right|_{t<-\delta}=\{(t,-t \bar{\omega}, r, r \bar{\omega}): t<-\delta, r \in \mathbf{R} \backslash 0\},
\end{gathered}
$$

where now $\{(t,-t \bar{\omega})\} \subset\{x=0\}$. Note that $B^{\bar{\omega}}$, the tangent plane to char $\square$ at $\pm(1, \bar{\omega})$, is $\left\{\tau=\eta_{1}\right\}$ and hence $\pi B^{\bar{\omega}} \subset\left\{\tau^{2} \leq|\eta|^{2}\right\}$, a set disjoint from the hyperbolic region. (When $n=2, \pi B^{\bar{\omega}} \subset\left\{\tau^{2}=\left|\eta^{2}\right|\right\}$.) In view of the role played by $B^{\check{\omega}}$ in $\S 4$, this suggests that anomalous singularities due to the selfspreading of a gliding ray may be significantly weaker than in the transversal case, perhaps only of strength $\sim 3 s-n$. (This observation is due to M. Reed.)

5.2. Crossing of two gliding rays. Let $\omega_{1}=(0,1,0), \omega_{2}=(0,-1,0)$ and in (5.2) replace the hypothesis on $W F_{b} u$ by

$$
\left.W F_{b} u\right|_{t<-\delta}=\bigcup_{i=1,2}\left\{\left(t,-t \omega_{i}, r, r \omega_{i}\right): t<-\delta, r>0\right\}
$$

Now $\pi \stackrel{\circ}{B}^{\omega_{1}, \omega_{2}}$ is a (2-dimensional) subset of $\left\{\tau^{2} \geq|\eta|^{2}\right\}$, so considering the arguments of $\S 3$, one might expect anomalous singularities of strength $\sim 2 s-$ $n / 2$ to appear in $x>0$. However, Remark 3.36 and the proof of Lemma 3.18 suggest, if gliding rays are thought of as limits of transversal rays, that cancellations could render these singularities significantly weaker.

Note added in proof. The author has recently shown that in both of problems 5.1 and 5.2, anomalous singularities of strength $2 s-n / 2+2+\varepsilon$ appear in $x>0$.

\section{REFERENCES}

1. M. Beals, Spreading of singularities for a semilinear wave equation, Duke Math. J. 49 (1982), 275-286.

2. __ Self-spreading and strength of singularities for solutions to semilinear wave equations, Ann. of Math. 118 (1983), 187-214.

3. __ Propagation of smoothness for nonlinear second-order strictly hyperbolic differential equations, Proc. Sympos. Pure Math., vol. 43, Amer. Math. Soc., Providence, R.I., 1985, pp. 21-44.

4. M. Beals and G. Metivier, Progressing wave solutions to certain nonlinear mixed problems, Duke Math. J. 53 (1986), 125-137.

5. __ Reflection of transversal progressing waves in nonlinear strictly hyperbolic mixed problems, Amer. J. Math. 109 (1987), 335-359.

6. J. Chazarain and A. Piriou, Introduction to the theory of linear partial differential equations, North-Holland, Amsterdam, 1982. 
7. F. David and M. Williams, Singularities of solutions to semilinear boundary value problems, Amer. J. Math. 109 (1987), 1087-1109.

8. L. Hörmander, The analysis of linear partial differential operators. III, Springer-Verlag, Berlin, 1985.

9. R. B. Melrose and J. Sjöstrand, Singularities of boundary value problems. II, Comm. Pure Appl. Math. 35 (1982), 129-168.

10. G. Metivier, Propagation, interaction, and reflection of discontinuous progressing waves for semilinear hyperbolic systems, preprint, 1986.

11. J. Rauch, Singularities of solutions to semilinear wave equations, J. Math. Pures Appl. 58 (1979), 299-308.

12. J. Rauch and M. Reed, Nonlinear microlocal analysis of semilinear hyperbolic systems in one space dimension, Duke Math. J. 49 (1982), 397-475.

13. M. Sablé-Tougeron, Régularité microlocale pour des problèmes aux limites non linéaires, Ann. Inst. Fourier (Grenoble) 36 (1986), 39-82.

14. M. Williams, Spreading of singularities at the boundary in semilinear hyperbolic mixed problems I: Microlocal $H^{s, s^{\prime}}$ regularity, Duke Math. J. 56 (1988), 17-40.

Department of Mathematics, University of North Carolina, Chapel Hill, North CarOLINA 27514 